\title{
DEMOCRACIA INTERNACIONAL, DERECHO HUMANO A LA DEMOCRACIA E INTERVENCIÓN HUMANITARIA*
}

\author{
Hugo Omar Seleme \\ CONICET Universidad Nacional de Córdoba
}

RESUMEN. El objetivo que persigue este trabajo es cuestionar la existencia de un Derecho Humano a la Democracia cuyos titulares son todos los seres humanos. Adicionalmente pretende mostrar que lo que en realidad existe es un derecho a la Democracia Internacional cuyos titulares son los Estados legítimos. Si el argumento que se ofrece para justificar este derecho es correcto, entonces se sigue una importante consecuencia con respecto a la institución de la intervención humanitaria. Específicamente, aparece un nuevo mandato democratizador. Uno que no requiere democratizar las instituciones estatales sino las instituciones internacionales.

Palabras clave: legitimidad, coacción, sistema internacional de estados.

ABSTRACT. The objective of this paper is to question the existence of a Human Right to Democracy whose holder is every human being. Additionally it aims to show that there actually is an International Right to Democracy whose holders are legitimate states. If the argument offered to justify this right to International Democracy is correct then an important implication regarding the institution of humanitarian intervention follows. Specifically, a new democratizing mandate appears. One that does not require to democratize the domestic institutions but the international ones.

Keywords: legitimacy, coercion, international state system.

* Fecha de recepción: 7 de julio de 2012. Fecha de aceptación: 15 de noviembre de 2012. 


\section{INTRODUCCIÓN}

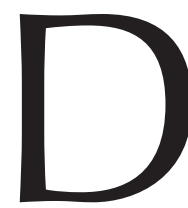

esde el final de la Guerra Fría la opinión de que existe un Derecho Humano a la Democracia comenzó a ganar fuerza entre los filósofos políticos y los doctrinarios del derecho internacional ${ }^{1}$. Tal posición tiene profundas consecuencias prácticas si es puesta en combinación con otra generalmente aceptada desde el final de la Segunda Guerra Mundial que afirma que la violación masiva de derechos humanos por parte de un Estado justifica la intervención armada o humanitaria con el objetivo de poner fin a tal trasgresión ${ }^{2}$. La justificación jurídica de este tipo de intervención ha sido disputada en el ámbito internacional, debido a que la Carta de las Naciones Unidas sólo contempla la intervención que tiene por objeto poner fin a potenciales amenazas para la paz (art. 7). No obstante, tanto a nivel filosófico ${ }^{3}$ como a nivel político ${ }^{4}$ su aceptación es amplia.

La consecuencia que se sigue de estas dos posiciones es que está justificado que un Estado intervenga en otro que no posee una organización política democrática con el objeto de favorecer su instauración ${ }^{5}$. Que esta conclusión se siga de premisas ampliamente aceptadas seria sólo una cuestión de interés teórico si no fuese porque una

1 Entre los filósofos políticos puede mencionarse a F. TESÓn (1998) y A. BUCHANAN (2004). Entre los juristas internacionales se encuentra G. FOX (1992) y Th. FrANCK (1992). De igual manera, el Comité de derechos humanos ha interpretado que la Convención sobre Derechos Civiles y Políticos establece un Derecho Humano a la Democracia. Aunque el texto de la Convención fue confeccionado de modo de dar cabida a sistemas electorales no competitivos tales como los que existían en el bloque soviético, con la caída de la URSS quedó despejado el camino para interpretarla como estableciendo un Derecho Humano a la Democracia.

${ }^{2}$ Este es un concepto restringido de intervención humanitaria que las circunscribe al caso de intervenciones armadas. Una utilización del mismo puede encontrarse en VINCENT (1974: 8). El concepto amplio, por el contrario, considera intervención humanitaria a cualquier injerencia en los asuntos internos de otro Estado con el objeto de proteger los derechos humanos. Medidas económicas tales como el embargo o el otorgamiento de incentivos serían tipos de intervención humanitaria. Una utilización del concepto amplio de intervención humanitaria puede encontrarse en BEITZ (1979-1999: 73-75) y en MOELLENDORF (2002: 117-119).

He optado por utilizar el concepto restringido porque representa el caso más claro y extremo. Si uno opta por el concepto restringido de intervención humanitaria el caso a favor de la misma consiste en mostrar que existe un supuesto de guerra justa. Es decir, la institución de la intervención humanitaria retorna a un estadio previo a la paz de Westfalia con relación a la guerra justa. Luego de Westfalia el único motivo de guerra justa fue la auto-defensa. La intervención humanitaria establece causales de guerra justa más amplias que la mera auto-defensa, a saber, la protección de los derechos humanos en un país extranjero. Esta ampliación de las causales de guerra justa parece revertir el movimiento a favor de restringirlas que se iniciase con VITORIA, SUÁREZ y continuase con GROCIO. No obstante puede apreciarse en los últimos años un intento por restringir las causales de intervención humanitaria. Un caso claro en este sentido es D. Luban quien ha corregido algunas de las posiciones que sostenía en trabajos previos volviendo las condiciones de la intervención humanitaria mucho más exigentes de lo que antes consideraba (LuBAN, 2002).

3 Uno de los primeros en defender esta postura ha sido D. Luban (1980). Básicamente LuBAN sostenía que la intervención en un país extranjero con el objeto de defender los derechos humanos se encontraba justificada aunque violase la soberanía y pudiese calificarse según el orden legal internacional como una agresión.

${ }^{4}$ La mayor muestra de que existe aceptación política en el ámbito internacional respecto de la intervención humanitaria puede encontrase en la resolución 60/1 de la Asamblea General de las Naciones Unidas, dictada el 24 de octubre del 2005, por la cual aprobó el informe de la International Comission on Intervention and State Sovereignty.

${ }^{5}$ Entenderé que un sistema es democrático cuando cada uno de los ciudadanos posee un derecho igual a expresar su opinión a través del voto adoptándose aquella decisión que obtiene algún tipo de mayoría. El concepto que tengo en mente es, como puede apreciarse, uno meramente de índole procedimental. Por supuesto, existen concepciones más robustas de democracia pero a los fines de este trabajo conviene adoptar un concepto mínimo. 
potencia militar de la envergadura de Estados Unidos, ha hecho de tal mandato democratizador uno de los ejes de su política exterior. Al respecto señalaba G. W. BusH en su discurso sobre el Estado de la Unión del 20 de junio de 2004:

[...] (W)e will finish the historic work of democracy in Afghanistan and Iraq, so those nations can light the way for others and help transform a troubled part of the world [...] America is a nation with a mission, and that mission comes from our most basic beliefs. We have no desire to dominate, no ambitions of empire. Our aim is a democratic peace, a peace founded upon the dignity and rights of every man and woman. America acts in this cause with friends and allies at our side, yet we understand our special calling: This great republic will lead the cause of freedom (Bush, 2004).

El justificar las intervenciones armadas en base a la promoción y defensa de la democracia no es algo que sea nuevo en la política estadounidense. La justificación ofrecida por W. WILSON para involucrar a Estados Unidos en la Primera Guerra Mundial y la ofrecida luego por F. RoosvelT para tomar parte en la Segunda Guerra Mundial, ya apelaban a la promoción de la democracia ${ }^{6}$. La novedad reside en que ahora se utiliza como justificación de la intervención a un tipo especifico de estándar normativo: un Derecho Humano a la Democracia. La alegada existencia de un Derecho Humano a la Democracia, permite presentar las intervenciones armadas con el objeto de promover o proteger esta forma de gobierno como un caso de intervención humanitaria.

El objetivo que persigue este trabajo es cuestionar la existencia de un Derecho Humano a la Democracia cuyos titulares son todos los seres humanos. Adicionalmente pretende mostrar que lo que en realidad existe es un derecho a la Democracia Internacional cuyos titulares son los Estados legítimos. Si el argumento que se ofrece para justificar este derecho es correcto, entonces se siguen importantes consecuencias con respecto a la institución de la intervención humanitaria.

La estructura del trabajo es la siguiente. En la sección I mostraré en donde radica el atractivo de presentar a las intervenciones democráticas como intervenciones de índole humanitaria. En la sección II presentaré una concepción de los derechos humanos que permite justificar que los Estados garanticen su satisfacción por parte de otros Estados, aun recurriendo a la intervención armada. En la sección III mostraré que si se adopta esta concepción no puede sostenerse que exista un Derecho Humano a la Democracia ${ }^{7}$. Lo que existe es un derecho a que el esquema de instituciones interna-

${ }^{6}$ Wilson en su War Message to the Congress del 2 de abril de 1917 señalaba: «...(W)e shall fight for the things which we have always carried nearest our hearts - for democracy, for the right of those who submit to authority to have a voice in their own governments, for the rights and liberties of small nations, for a universal dominion of right by such a concert of free peoples as shall bring peace and safety to all nations and make the world itself at last free...» y agregaba luego, en un pasaje que el discurso de G. BusH antes transcripto parece replicar como un eco: «...The world must be made safe for democracy. Its peace must be planted upon the tested foundations of political liberty. We have no selfish ends to serve. We desire no conquest, no dominion...» (WILSON, 1917). Roosevelt, por su parte señalaba: «Democracy's fight against world conquest is being greatly aided, and must be more greatly aided, by the rearmament of the United States and by sending every ounce and every ton of munitions and supplies that we can possibly spare to belp the defenders who are in the front lines...». Y agregaba luego: «We must be the great arsenal of democracy...» (ROOSEVELT, 1940).

7 La discusión que me interesa abordar es de moralidad política. La pregunta relevante, entonces, es la de si existen razones morales que justifiquen un Derecho Humano a la Democracia. Las razones de otra índole, como aquellas referidas a la estabilidad política, no son aquí relevantes a menos que se pruebe su carácter moral. Del mismo modo son irrelevantes aquí los argumentos en contra de las transiciones democráticas basadas en la incerteza acerca de los resultados que puedan producir. Los datos ofrecidos por E. MANSFIELD y J. 
cionales posea un mecanismo democrático de toma de decisiones, esto es un derecho a la Democracia Internacional. En la sección IV me encargaré de mostrar las consecuencias que trae aparejada para la intervención humanitaria la concepción de derechos humanos presentada y la existencia de un derecho a la Democracia Internacional. Adicionalmente, será mostrado que la concepción no sólo posee en mayor medida algunos de los atractivos que traía aparejada la supuesta existencia de un Derecho Humano a la Democracia, sino que posee otros de mayor o igual envergadura.

\section{EL ATRACTIVO DE LA INTERVENCIÓN HUMANITARIA FUNDADA EN UN DERECHO A LA DEMOCRACIA}

Una de las principales objeciones que se ha dirigido a la política exterior de Estados Unidos de intervenir en otros Estados con el objeto de instaurar regímenes democráticos, sostiene que se trata de un intento de imponer coercitivamente a otros pueblos valores occidentales o estadounidenses ${ }^{8}$. La forma de gobierno democrática es valiosa para los estadounidenses, señala la objeción, e intentar imponerla a otros pueblos no está más justificado de lo que estaría a nivel doméstico el intento de imponer los propios valores personales a otros ciudadanos. Se trata, continúa la objeción, de un caso de doble estándar. Mientras a nivel domestico se sostiene que no esta justificada la coacción para imponer los propios valores a otros ciudadanos, puesto que el uso de la coacción debe justificarse sobre la base a valores o principios que aquel que va a ser

SNYDER (2005) señalando que los regímenes que están en transición hacia la democracia son más beligerantes que los regímenes autocráticos estables, no son dirimentes para el objetivo que persigo. La pregunta por si existe un Derecho Humano a la Democracia puede distinguirse de la pregunta acerca de en qué circunstancias es razonable adoptar políticas para promover regímenes democráticos.

${ }^{8}$ Otra acusación de la que han sido objeto las intervenciones estadounidenses es la de perseguir objetivos económicos o políticos que son enmascarados detrás de la supuesta promoción de la democracia. S. W. HoOK afirma: «The U.S. pursuit of global democratization historically has served an ambiguous combination of altruistic aspirations and concrete national self-interest. Unfortunately, the subordination of U.S. democratic ideals to geopolitical concerns during the Cold War... fuelled ideological polarization within many developing countries and left a legacy of mistrust and resentment that has yet to be overcome more than a decade after the Cold War's collapse...» (HOOK, 2002: 109). Uno de los primeros en formular esta línea de crítica fue N. CHOMSKY (1992).

Como un ejemplo patente de una intervención armada cuyo objetivo fue encubrir la persecución de otros intereses, algunos autores citan el caso de la guerra de Irak. Estados Unidos apoyó el régimen autocrático mientras sirvió a sus propósitos económicos, y lo atacó cuando este no fue más el caso. Afirma T. COFMAN WITTE: «Washington's support of Saddam Hussein's Iraq during his long war with Iran served America's interest in stability, because Saddam's onslaught prevented the revolutionary Islamism of Khomeini's Iran from extending its influence further into the gulf, a move that would have undermined the stability of Saudi Arabia and other oilproducing states. When Saddam Hussein gassed his own population in the midst of that conflict, America's official response was mild. Only when Hussein turned his revisionist ambitions on American allies Kuwait and Saudi Arabia, threatening global energy supplies, did the United States turn against him» (WITTES, 2008: 17).

Una objeción emparentada critica la utilización de un doble estándar por parte de la política exterior estadounidense. A diferencia de la que he señalado en el texto, esta sostiene que no todos los países son medidos por Estados Unidos utilizando la misma métrica democrática a la hora de decidir la intervención. Específicamente, durante la guerra fría y la lucha en contra de comunismo fueron tolerados regímenes autoritarios si esto era funcional a la lucha anti-comunista. La tolerancia de Estados Unidos, o el abierto apoyo, del Sha en Irán, del general Anastasio Somoza en Nicaragua, de Ferdinand Marcos en Filipinas o de las dictaduras militares latinoamericanas, son ejemplos de esta política de doble estándar. El ejemplo más reciente y patente de doble estándar es el modo en que Estados Unidos ha conducido sus relaciones con China, pasando por alto el déficit democrático de su gobierno y la violación de derechos humanos (NEIER, 1997). 
coaccionado pueda aceptar ${ }^{9}$, a nivel internacional se utiliza la coacción para imponer a otros pueblos la forma de gobierno que los estadounidense consideran valiosa.

El modo más directo de enfrentar esta objeción es sostener — como señala G. BusH en el discurso antes citado- que la democracia se encuentra fundada en la dignidad y los derechos que todo ser humano posee. Si la democracia es un derecho humano, las intervenciones armadas con el objeto de protegerla no son un intento de imponer un valor o forma de gobierno propia de un pueblo a otros, sino un intento de proteger los derechos que todos los individuos poseen, sea cual sea el pueblo al que pertenecen. Adicionalmente, puesto que la práctica de la intervención humanitaria es ampliamente aceptada a nivel internacional, lo mismo debería suceder con las intervenciones que tienen por objeto proteger o promover formas de gobierno democráticas. Las quejas de aquellos que se oponen a las intervenciones con el objeto de establecer sistemas democráticos son transformadas en intentos de defender un orden internacional donde los Estados no poseen ningún límite en el modo en que pueden tratar a sus ciudadanos. Si existe un Derecho Humano a la Democracia, la razón de principio para cuestionar las intervenciones democráticas debe ser que se cuestiona en general la intervención humanitaria.

En consecuencia, el primer atractivo de considerar que existe un Derecho Humano a la Democracia y de equiparar las intervenciones con el objeto de protegerlo a intervenciones humanitarias, es de índole política. Permite justificar un principio de política exterior que Estados Unidos ha seguido desde el siglo pasado. Si la intervención humanitaria se encuentra moralmente justificada, entonces también lo está la política exterior estadounidense que ha tenido por objeto imponer a otros pueblos su forma de gobierno ${ }^{10}$. El caso a favor de la intervención democrática se vuelve tan fuerte como el caso a favor de la intervención humanitaria. La existencia de un Derecho Humano a la Democracia permite que la fuerza de los argumentos justificatorios a favor de la intervención humanitaria se transmita a la justificación de la intervención democrática.

Ahora bien, el vincular la intervención humanitaria con la intervención democrática no solo fortalece el caso de esta última. Que exista un Derecho Humano a la Democracia también fortalece la justificación de la intervención humanitaria, al permitir descartar una de las objeciones más potentes que contra ella se ha dirigido. Este es el segundo de los atractivos que posee la posición que sostiene que existe un Derecho Humano a la Democracia.

Aunque la práctica de la intervención humanitaria es ampliamente aceptada, ha sido objeto de una crítica poderosa. El poder de la misma radica tanto en el peso del argumento, como en el peso político del Estado que la ha formulado. La posición del gobierno chino con relación a la intervención humanitaria sostiene que la misma se funda en una errónea concepción de los derechos humanos. Según la correcta interpretación de los derechos humanos, continua la objeción, la trasgresión de estos de-

\footnotetext{
9 Esta exigencia se funda en el deber de civilidad (RAWLS, 1993: 217).

10 Lo señalado sólo cuenta como un atractivo si uno esta interesado en justificar las políticas estadounidenses. Sin embargo, si uno tiene en mente, por un lado, que la mayoría de los filósofos políticos residen en Estados Unidos o en países que apoyan su política exterior, y por el otro, que uno de los objetivos de la Filosofía Política es reconciliarnos con nuestras propias instituciones, se entiende porque la mayor parte de los filósofos políticos puede haber considerado a esto un atractivo.
} 
rechos por parte de un Estado no es una razón que justifique la intervención de otros Estados en sus asuntos internos ${ }^{11}$. Los derechos humanos imponen deberes sobre los Estados pero su trasgresión no confiere al resto de los miembros de la comunidad internacional un derecho a intervenir. La razón de ello radica en que cualquier intervención vulneraría el derecho al auto-gobierno, que es uno de los valores centrales del derecho internacional ${ }^{12}$.

La posición del gobierno chino se funda en la idea socialista de que el Estado corporiza los intereses del pueblo y, por tanto, no puede existir conflicto de intereses entre ciudadanos y Estado o entre derechos humanos y soberanía que sirva para justificar la intervención extranjera (KENT, 1993: 21-22). Sin embargo, la objeción —una vez se la independiza de su base ideológica - apunta a un problema más general ${ }^{13}$. Cualquier tipo de intervención humanitaria implica una vulneración al derecho al auto-gobierno y, por tanto, debe ser defendida apelando a dos estrategias igualmente endebles: cuestionar que el auto-gobierno sea un valor; o sostener que cuando existe una violación masiva de derechos humanos el subsanar este mal tiene mayor importancia que el respecto irrestricto por el auto-gobierno ${ }^{14}$. Ambas estrategias parecen poco atractivas porque implican o bien abandonar un valor firmemente arraigado en el derecho internacional, o bien conceder que en cada intervención humanitaria existe un residuo moral provocado por la vulneración del derecho al auto-gobierno, aun si lo que se busca es promover o proteger valores de mayor importancia.

Ahora bien, si uno quiere mantener que el auto-gobierno es valioso y que no es el caso que toda intervención humanitaria implique su vulneración, una tercera estrategia queda disponible. Debe mostrarse que al menos existe un caso en que la intervención humanitaria no vulnera el derecho al auto-gobierno. Si existe un Derecho Humano a la Democracia, la intervención humanitaria con el objeto de promover o proteger esta forma de gobierno seria ese supuesto. La intervención humanitaria democrática no caería presa de la objeción fundada en la vulneración del auto-gobierno. Esto bastaría para mostrar que es falso que toda intervención humanitaria se encuentra en tensión con el auto-gobierno.

Tres razones determinan que, si existe un Derecho Humano a la Democracia, la intervención humanitaria para garantizarlo no quede sujeta a la objeción. En primer

11 Para una evaluación crítica de esta concepción de los derechos humanos, vid. BEITZ, 2009: 123-124.

12 Haciendo referencia a la importancia del valor del auto-gobierno o auto-determinación en el ámbito internacional, la Recomendación General 21/96 del Comité de derechos humanos sostiene en su art. 2: «The right to self-determination of peoples is a fundamental principle of international law. It is enshrined in article 1 of the Charter of the United Nations, in article 1 of the International Covenant on Economic, Social and Cultural Rights and article 1 of the International Covenant on Civil and Political Rights, as well as in other international buman rights instruments. The International Covenant on Civil and Political Rights provides for the rights of peoples to self-determination besides the right of ethnic, religious or linguistic minorities to enjoy their own culture, to profess and practise their own religion or to use their own language».

13 Un modo alternativo de presentar esta objeción en contra de la intervención humanitaria consiste en trazar una analogía entre la autonomía individual y la autonomía de los Estados. I. KANT, E. DE VATTEL y Ch. WOLFF son algunos de los pensadores que apelaron a esta analogía para fundar el derecho de los estados a la no-intervención.

${ }^{14}$ Uno de los que ha defendido esta posición es D. MollendDorf (2002). En su opinión la intervención humanitaria debe tener por objeto proteger o promover la justicia de la estructura básica. El autogobierno encuentra su límite en la injusticia. Señala al respecto: «...the right to share a common life together cannot trump other considerations of justice...» (MOLLENDORF, 118: 2002). 
lugar, porque el derecho humano al auto-gobierno pasa a ser entendido como un derecho referido a los individuos y no a los Estados. Este primer paso rompe la equivalencia entre el interés en el auto-gobierno protegido por los derechos humanos y el derecho de los Estados a la no-intervención en sus asuntos internos ${ }^{15}$. En segundo lugar, porque si el interés que los individuos tienen en el auto-gobierno sólo se satisface en el seno de instituciones democráticas, la intervención armada con el objeto de instaurar un sistema democrático no vulnera el interés en el auto-gobierno protegido por los derechos humanos. Al no existir democracia en el Estado intervenido, no existe un esquema de auto-gobierno que la intervención pueda vulnerar. Tercero, porque el objeto de la intervención es la instauración de un sistema democrático de gobierno que permita satisfacer el derecho al auto-gobierno ${ }^{16}$.

Uno de los más fervientes promotores y defensores de la política estadounidense de intervenciones democráticas, J. MURAVCHIK, ha expresado el argumento antes esquematizado de modo contundente. Intentando defender las intervenciones democráticas, señala en Exporting Democracy:

[...] The reason it is wrong to impose something on others, presumably, is because it violates their will. But, absent democracy, how can their will be known? Moreover, why care about violating people's will unless one begins with the democratic premise that popular will ought to be sovereign? (MURAVCHIK, 1991:34).

En síntesis, presentar a las intervenciones democráticas como un tipo de intervención humanitaria parece tener beneficios a dos puntas. Por un lado, fortalece el caso a favor de las intervenciones democráticas. Estas no son intentos de coaccionar a otros pueblos para que acepten valores propios de occidente, sino un modo de garantizar derechos humanos que todo individuo posee más allá del pueblo al que pertenece. Por otro, fortalece el caso a favor de la intervención humanitaria. No es el caso que toda intervención humanitaria implique una vulneración al derecho al auto-gobierno. La intervención democrática es un supuesto de intervención humanitaria donde tal vulneración no está presente.

\section{LOS DERECHOS HUMANOS COMO REQUERIMIENTOS DE LEGITIMIDAD}

Para determinar si existe un Derecho Humano a la Democracia es necesario articular previamente una concepción de los derechos humanos. Antes de avanzar sobre los detalles de la concepción que voy a presentar, puede ser de utilidad presentar la idea central. Según esta concepción los derechos humanos son las exigencias morales que aparecen debido a dos rasgos del orden internacional: su carácter coercitivo y su carácter estatal. El orden internacional impone de modo coercitivo sobre todos los individuos que habitan el planeta un sistema de Estados. A su vez, cada Estado está constituido por un conjunto de instituciones que se aplican de modo coercitivo. Que

${ }^{15}$ Esta equiparación entre ambos derechos es denunciada como algo peligroso, entre otros, por J. DoNELLI (1989: 148).

${ }^{16}$ Esta última razón muestra que la intervención democrática no solo no vulnera el derecho humano al auto-gobierno sino que tiene por objeto protegerlo. 
los derechos humanos sean la contrapartida del carácter coercitivo del orden internacional, y que existan dos niveles de coacción —el internacional y el estatal - es lo que explica uno de los rasgos centrales de los derechos humanos, a saber, que los Estados sean los principales responsables de su satisfacción y que la comunidad internacional aparezca como garante. El recurso extremo que posee la comunidad internacional para efectivizar esta garantía es el de la intervención humanitaria.

Para desarrollar esta concepción de los derechos humanos llevaré adelante los siguientes pasos. En primer lugar, en el apartado 2.1, explicitaré en qué sentido tanto las instituciones domésticas como las internacionales poseen carácter coactivo. En el apartado 2.2 argumentaré para mostrar que la existencia de un esquema institucional coercitivo genera exigencias de legitimidad política. Presentaré una concepción institucional de la legitimidad que la vincula con el carácter de autor que deben poseer los sujetos a quienes las instituciones se aplican. En los apartados 2.3 y 2.4 me detendré a señalar el conjunto de condiciones que deben satisfacer las instituciones domésticas y las internacionales, dado el diferente modo en que son coercitivas, para ser legítimas. Los derechos humanos aparecerán como las exigencias que las instituciones estatales deben satisfacer para ser legítimas ${ }^{17}$.

A partir de la concepción de derechos humanos elaborada, extraeré en la sección III las conclusiones que se siguen con respecto a la existencia de un Derecho $\mathrm{Hu}$ mano a la Democracia. Por un lado, mostraré que dado que puede existir legitimidad o auto-gobierno sin democracia, el derecho a la democracia no es uno que deba ser satisfecho por las instituciones domésticas ni —en consecuencia- garantizado por las instituciones internacionales. Sin embargo, por otro lado, mostraré que dentro de los derechos que el orden internacional debe conferir a los Estados para ser legítimo se encuentra el derecho a la democracia. El derecho a la democracia será presentado como una condición de legitimidad del orden internacional, no de las instituciones estatales. La conclusión será que aunque no existe un Derecho Humano a la Democracia cuyos titulares son los ciudadanos, sí existe un derecho a la Democracia Internacional cuyos titulares son los Estados.

\subsection{El carácter coactivo de las instituciones estatales e internacionales}

Dos rasgos de las instituciones determinan su carácter coercitivo. En primer lugar, la existencia de un esquema institucional debe volver más probable que un individuo opte por cierto curso de acción o soporte un determinado estado de cosas. Un modo en que algunas instituciones producen este efecto sobre las probabilidades es a través de la imposición de sanciones a la no realización de ciertas acciones o la utilización de la fuerza o la amenaza del uso de la fuerza para que determinado estado de cosas se materialice. No obstante, este no es el único modo en que un esquema incide sobre las probabilidades de que un individuo siga un curso de acción o soporte un estado de

17 Ch. BeitZ (2009) ha sido el primero en construir un modelo de derechos humanos haciendo referencia a estos dos niveles de responsabilidad. La concepción de derechos humanos que voy a presentar en el texto va más allá de la suya en dos sentidos. En primer lugar, vincula los dos niveles de responsabilidad con los diferentes modos en que el esquema de instituciones domésticas e internacionales son coercitivas. En segundo lugar, vincula a los derechos humanos con las exigencias de legitimidad política o autoría. 
cosas, con independencia de cuál sea su voluntad. Así, por ejemplo, el modo en que un esquema institucional recompensa los talentos naturales, o la manera en que distribuye el ingreso o las oportunidades, determinan que sea más probable para un individuo, por ejemplo, elegir una carrera en lugar de otra —optar por cierto curso de acción-o no disponer de ciertos recursos o bienes - soportar un estado de cosas-.

Este modo de concebir el carácter coactivo de un esquema institucional se aparta de la idea tradicional según la cual una regla o esquema institucional posee este carácter sólo cuando su cumplimiento se encuentra respaldado por la amenaza de una sanción o por el uso de la fuerza. La concepción tradicional entiende a la coerción como interferencia ${ }^{18}$. La razón de ello radica en que la amenaza de sanción o el uso de la fuerza aumentan las probabilidades de que el agente adopte un curso de acción con independencia de cuál sea su voluntad. No obstante, si es este efecto sobre las probabilidades el que hace que un esquema respaldado por la amenaza de sanción o el uso de la fuerza cuente como coactivo, no existe razón para sostener que normas o esquemas institucionales que producen idéntico efecto aun sin estar respaldadas por la amenaza de sanción o el uso de la fuerza, no son coactivas. La existencia de normas que recompensan la posesión y el desarrollo de ciertos talentos naturales, o que distribuyen los recursos y las oportunidades, inciden sobre la probabilidad de que un individuo adopte ciertos cursos de acción o soporte determinados estados de cosas, del mismo modo en que lo hacen las normas respaldadas por el uso de la fuerza o la amenaza de sanción.

El segundo rasgo de un esquema institucional que determina que sea coercitivo consiste en que éste no sólo produce el efecto antes señalado sino que persigue el efecto de aumentar la probabilidad de un curso de acción o el acaecimiento de un estado de cosas sobre ciertos individuos ${ }^{19}$. El carácter intencional de la incidencia sobre el aumento de las probabilidades es lo que permite distinguir la idea de coacción de las meras externalidades negativas.

Para entender la distinción entre externalidad negativa y coacción puede ser de utilidad un ejemplo elaborado por NozICK ${ }^{20}$. Este nos propone imaginar una situación donde hay 26 mujeres (A, B, C,... Z) y 26 varones (A', B', C’... Z') con deseos de casarse.

18 A. RIPSTEIN señala como representante paradigmático de la concepción tradicional a S. MiLL. La coerción: «...interferes with a person's liberty, and does so by imposing a cost on that person that he or she would not otherwise have borne...» (RIPSTEIN, 2004). Ph. PETTIT, por su parte, traza el origen de la noción de libertad como no-interferencia que subyace a la concepción tradicional de coerción hasta Th. HOBBES (PETTIT, 2008; PetTit y LovetT, 2009: 15).

19 Con ligeras modificaciones he estado interpretando coacción del mismo modo que PETTIT utiliza el concepto de dominación. He decidido, no obstante, utilizar otra terminología por dos motivos. En primer lugar, no tengo claro si PETTIT afirmaría que puede existir dominación sin intención de dominar. Si este fuese el caso, su noción de dominación sería diferente de la noción de coacción que estoy utilizando, puesto que no puede existir coacción sin intención de coaccionar. PEтTiT simplemente señala: «One person, A, controls the choice of another person, $B$, when $A$ does something that has the intentional or quasi-intentional effect of raising the probability that B will choose according to A's taste or judgment — raising it beyond the probability that this would have had in A's absence...» (PETTIT, 2009: 42). Esto todavía no es concluyente respecto a si debe o no existir intención de dominar o controlar. En segundo lugar, mientras PETTIT piensa que la exigencia moral que engendra la posibilidad de que exista dominación es la de no-dominación o control, la coacción tal como quedará más claro en lo que sigue no engendra la exigencia moral de no-coacción sino la de autoría.

${ }^{20}$ Lo que señalo en estos párrafos acerca de la distinción entre externalidad y coacción ha sido desarrollado en un trabajo previo que aquí simplemente sigo (SELEME, 2010: 86-87). 
Cada uno de los individuos tiene el mismo orden de preferencias con relación a la lista de individuos del sexo opuesto. Cada uno prefiere en orden decreciente desde el individuo A o A' (según que sea varón o mujer) hasta el individuo $\mathrm{Z}$ o Z'. Todos los varones lo que más prefieren es casarse con $\mathrm{A}$ y luego sigue $\mathrm{B}$ y así hasta $\mathrm{Z}$. Todas las mujeres a quien más prefieren es a A' y luego a B' y así hasta Z'. Supongamos que A y A' se casan, esto aumenta la probabilidad de que los otros individuos adopten un curso de acción —es ahora más probable que adopten el segundo individuo en la lista de los que más desean puesto que los primeros ya se han casado entre sí- pero no por esto afirmamos que A y A' han coaccionado a los 50 individuos restantes. Ni siquiera lo afirmamos en el caso extremo donde todos, salvo Z y Z', ya se han casado. Aunque no tienen más que una opción para elegir, aunque el casamiento de los otros ha aumentado las probabilidades de que se elijan recíprocamente, no pensamos por ello que hayan sido coaccionados (Nozick, 1974: 263). Lo que determina que aquí no haya coacción es que cada uno de los individuos que elige su pareja no tiene la intención de incidir sobre los posibles cursos de acción de los demás. Su única intención es casarse con quien ha elegido. La incidencia sobre el aumento de la probabilidad de que otro adopte cierto curso de acción —el de elegir a la persona que sigue en su lista de preferencias- es una mera externalidad.

Si variamos el ejemplo, puede verse que lo relevante se encuentra en la intención. Imaginemos ahora que las preferencias que cada individuo perteneciente al grupo $\mathrm{A}$, B... salvo Z se refiere a que el resto no se case con A', B' y así en orden decreciente. Z, por su parte, tiene las mismas preferencias que en caso anterior. Él prefiere casarse con A', luego le sigue B' y así en orden decreciente. A', B'... Z' prefieren casarse, aunque les es indistinto con quien y lo harán con cualquiera que se lo pida. En este caso imaginemos que A y A' se casan. A porque lo que más desea es que ninguno de los otros se case con A' y casarse con ella es un modo de evitar que los otros puedan hacerlo. A' porque le es indistinto con quien casarse y A fue el primero en ofrecérselo. Luego le toca el turno a B que elige a B' y así sucesivamente hasta llegar a Z. Cuando Z elige sólo le queda una opción disponible Z', que era la pareja que menos deseaba. Todos desde A hasta $\mathrm{Y}$ han tenido la intención de evitar que $\mathrm{Z}$ se casase con alguien, $\mathrm{A}$ tenía la intención de que no se casase con A', B con B' y así sucesivamente. ¿'Puede Z quejarse de algo en esta nueva situación? Pienso que sí. Todos han incidido de modo intencional en el modo en que $\mathrm{Z}$ conduce su existencia. Todos han intentado determinar a quien elige como pareja. En este sentido, puede afirmarse que $\mathrm{Z}$ ha sido coaccionado ${ }^{21}$. La acción de cada uno de los otros ha tenido el efecto intencional de aumentar las probabilidades de que $\mathrm{Z}$ adopte un curso de acción ${ }^{22}$.

Que el aumento de la probabilidad sea ahora intencional es lo que nos inclina a pensar que en estos casos ha existido algún tipo de coacción. La situación tiene la misa estructura que la que se presenta cuando se utiliza la amenaza o la fuerza. Si A, B... Y, para evitar que otros se casen con A', B'... Z', formulan la amenaza de que si otro lo

${ }^{21}$ Los miembros del grupo A'...Z' no han coaccionado a Z. Aun si han contribuido a restringir sus opciones, este no ha sido un efecto que buscasen intencionalmente.

${ }_{22}$ Me he concentrado en el caso de $\mathrm{Z}$ porque es en quien la coacción se percibe con mayor fuerza. No obstante, salvo A el resto de los individuos en grado creciente se encuentran coaccionados. Las acciones de otros han aumentado de modo intencional la probabilidad de que adopten un curso de acción, a saber, no casarse con las parejas que los demás han elegido. 
hace recibirá un castigo o ponen obstáculos materiales para que el casamiento pueda celebrarse, lo que han hecho ha sido elevar de modo intencional las probabilidades de que otros no tomen este curso de acción. Lo mismo que han hecho, en el caso antes descripto, al casarse con sus respectivas parejas. Lo que muestra el ejemplo, es que existe coacción cuando de modo intencional se incide sobre las probabilidades de que otro adopte un curso de acción o soporte un estado de cosas.

Trasladado a los esquemas institucionales, tenemos entonces que un esquema es coactivo no simplemente cuando incide sobre la probabilidad de que un agente elija un curso de acción o soporte un estado de cosas, sino adicionalmente cuando esa es su pretensión, cuando la incidencia en las probabilidades se trata de una consecuencia intencional. Esto nos deja con el problema de determinar sobre quienes recaen las consecuencias intencionales de un esquema institucional estatal.

Th. Pogge (1989: 45) ha trazado una distinción entre las consecuencias producidas intencionalmente por las instituciones y aquellas que no lo son ${ }^{23}$. Lo primero que POGGE distingue son las consecuencias que los esquemas institucionales producen - aquellos efectos que no sucederían en ausencia del esquema institucional-, de aquellas que meramente dejan acaecer — aquellos efectos que suceden con independencia de la existencia del esquema institucional - Las consecuencias producidas intencionalmente ${ }^{24}$ son un subconjunto de las consecuencias producidas. En particular, se trata de las consecuencias establecidas de modo directo por el esquema institucional a través de sus reglas. Por ejemplo, una consecuencia establecida por las reglas que crean una economía de mercado es que las personas puedan comprar y vender. Una consecuencia producida, pero no establecida sino meramente engendrada, del mismo conjunto de reglas es que algunas personas que no tienen nada de valor para ofrecer caigan por debajo de la línea de pobreza. Esta última consecuencia es producida - ya que estas personas presumiblemente no serían pobres si existiese otro conjunto de reglas que regulase la economía- pero no es establecida en tanto no hay ninguna regla que fije de modo explícito su nivel de ingresos.

Tomando la distinción de POGGE, puede señalarse que un esquema institucional pretende incidir sólo sobre los probables cursos de acción que adoptan o los estados de cosas que soportan aquellos a quienes establece como destinatarios. No pretende incidir sobre todos en quienes produce consecuencias. Las consecuencias que un esquema institucional previsiblemente engendra sirven para determinar a qué han sido coaccionados aquellos a quienes establece como destinatarios ${ }^{25}$. Así, por ejemplo, en el caso del esquema institucional que organiza la economía de mercado dentro de un

${ }^{23}$ Lo que digo aquí sobre las consecuencias intencionales de un orden institucional ha sido desarrollado en trabajos previos a los que aquí sigo con ligeras modificaciones (SELEME, 2007, 2009 y 2010).

${ }^{24}$ Estas consecuencias son las consideradas moralmente relevantes por las concepciones que adoptan un enfoque deontológico a la hora de evaluar diseños institucionales. Una concepción paradigmáticamente deontológica, en este sentido, es la de NozicK.

25 Esta distinción captura algo característico de la coacción. Mientras no puede ser el caso que un sujeto coaccione a otro sin intención, es posible que lo coaccione a adoptar un curso de acción o soportar un estado de cosas que excede la intención de quien coacciona. Si amenazo a alguien con la intención de que se haga un disparo en la cabeza a consecuencia del cual muere, sin duda lo he coaccionado. La intención cuenta a la hora de establecer si hubo o no coacción y quién es el sujeto coaccionado. Sin embargo, no cuenta para determinar a qué lo coaccioné. Lo he coaccionado a matarse, con independencia de que mi intención haya sido esa o no. La previsibilidad de la consecuencia, y no mi intención efectiva, es la que cuenta en este último caso. 
Estado, las personas a quienes se aplica coercitivamente serán quienes habitan el territorio del mismo, a quienes las normas establecen como destinatarios. La consecuencia engendrada de la pobreza, servirá para determinar a qué han sido coercionados algunos de estos ciudadanos. Si la pobreza, como una consecuencia engendrada, recae tanto sobre sujetos a quienes el esquema establece como destinatarios como sobre quienes no lo son, dado el carácter intencional de la coacción, sólo de los primeros puede afirmarse que de modo coactivo han sido puestos en una situación de pobreza. Los segundos son sólo víctimas de una externalidad negativa producida por el esquema institucional, pero no son sujetos a quienes el esquema institucional se aplica coactivamente. Así, si las instituciones que organizan la economía de mercado en un Estado tienen efectos colaterales en quienes habitan un Estado vecino - por ejemplo, alterando su nivel de ingreso debido al aumento o disminución de las exportaciones- los ciudadanos del segundo Estado no están siendo coercionados por un esquema institucional extranjero. Están sufriendo o disfrutando de sus consecuencias, pero en tanto aquel esquema no los establece como destinatarios, falta el requisito de la intencionalidad y, por tanto, no hay coacción ${ }^{26}$.

En síntesis, si un esquema institucional incide sobre las probabilidades que tienen de adoptar un curso de acción o soportar un estado de cosas aquellos a quienes establece como destinatarios, entonces tiene carácter coercitivo con relación a ellos. Para determinar a qué son coaccionados — esto es, para determinar el curso de acción o estado de cosas sobre cuya probabilidad incide el esquema institucional - lo relevante no sólo son las consecuencias establecidas sino también aquellas que previsiblemente engendra.

Tanto las instituciones domésticas como las internacionales son coercitivas en el sentido señalado. En ambos casos, su existencia aumenta la probabilidad de que los sujetos a quienes establecen como destinatarios adopten cierto curso de acción o soporten algún estado de cosas. En lo que se distinguen es en el tipo de sujeto a quienes establecen como destinatarios - esto es, a quienes coercionan - y el tipo de curso de acción o estado de cosas cuya probabilidad aumentan —esto es, a qué son coercionados-.

El sujeto paradigmático a quienes se aplican las instituciones domésticas estatales son las personas consideradas aisladamente, los individuos que habitan un determinado territorio. El esquema institucional que los establece como destinatarios aumenta la probabilidad de que cada ciudadano goce de ciertas expectativas vitales, derechos y recursos. Siguiendo con el ejemplo introducido previamente, el esquema institucional que establece a ciertos individuos como destinatarios contiene reglas que organizan el libre mercado, reconocen el derecho a la herencia, reconocen y protegen a la familia, y no fijan mínimos vitales, es probable que los individuos que nacieron en familias con

${ }^{26}$ En mi opinión esta es la noción rawlsiana de instituciones coercitivas. Que una institución se aplique coercitivamente no se encuentra vinculado al uso o a la amenaza de uso de la fuerza. No obstante soy consciente de que esta interpretación no es pacífica. Así, por ejemplo, M. BLAKE (2002) presupone en su argumento que RAWLS se encuentra comprometido con la idea tradicional de coacción.

G. COHEN (2000: 134-148) señala dos modos alternativos de interpretar en qué consisten las instituciones básicas según RAWLS, uno de los cuales es semejante a la idea de instituciones de aplicación coercitiva que presento aquí. COHEN sostiene que sea que uno opte por una noción restringida de estructura básica —coercitiva en el sentido tradicional — o una más amplia — coercitiva en el sentido que utilizo en el texto- la concepción rawlsiana se encuentra en problemas. 
altos recursos gocen de mayores oportunidades y expectativas vitales que aquellos que nacieron en una familia pobre. Es también probable que los individuos nacidos en familias pobres y que no poseen ninguno de los talentos naturales premiados por el mercado, no tengan ni siquiera los mínimos recursos para subsistir. De modo coercitivo, el esquema institucional fija las expectativas tanto de quienes favorece como de quienes perjudica. Aumenta la probabilidad de que acaezcan ciertos estados de cosas. Lo mismo puede decirse respecto del aumento de probabilidades de que algunos individuos adopten un curso de acción. Así, por ejemplo, en un esquema institucional como el presentado, es más probable que los individuos nacidos en familias con bajos recursos, menores oportunidades y expectativas vitales, opten por delinquir ${ }^{27}$.

Las instituciones internacionales se diferencian de las domésticas en dos sentidos. En primer lugar, establecen como destinatarios tanto a los individuos considerados aisladamente como a los individuos organizados políticamente, esto es, a los Estados. En segundo lugar, imponen cursos de acción o estados de cosas característicamente diferentes de los que imponen las instituciones domésticas ${ }^{28}$.

Por lo que respecta a la primera diferencia, las instituciones internacionales establecen un sistema de unidades políticas territoriales - los Estados- que poseen autoridad dentro de sus fronteras. A su vez, las reglas institucionales del esquema internacional establecen como destinatarios a los Estados. En este sentido son coercitivas tanto con relación a los individuos como con relación a los Estados. Establecen que los individuos habiten un sistema de Estados. Establecen que los Estados gocen de ciertos derechos, posean ciertos deberes, o soporten cierto estado de cosas.

Con respecto a la segunda diferencia, deben distinguirse los estados de cosas y cursos de acción que las instituciones internacionales imponen sobre los individuos, de aquellos que imponen sobre los Estados. Sobre estos últimos imponen un esquema de derechos y deberes. Así, por ejemplo, establecen que los Estados tienen derecho a disponer de los recursos naturales que se encuentran en su territorio, o poseen el deber de no intervenir en los asuntos de otros Estados, no llevar adelante guerras de agresión o respetar los tratados. Estas reglas poseen carácter coercitivo en tanto aumentan la probabilidad de que ciertos estados de cosas acaezcan a los Estados — por ejemplo, que unos dispongan de mayores recursos naturales que otros-o adopten ciertos cursos de acción - por ejemplo, que un Estado respete las decisiones que sobre cuestiones domésticas adopta otro-.

Por su parte, el orden internacional impone sobre los individuos un sistema de Estados, esto es, un sistema cuyas unidades — los Estados — son a su vez esquemas

${ }_{27}$ Sobre la correlación entre pobreza y delincuencia existen múltiples trabajos. Por todos, puede verse el de A. KARMEN (2000) En el mismo se muestra a partir de datos de la ciudad de Nueva York que las personas que habitan una situación de pobreza no sólo tienen más probabilidades de delinquir (KARMEN, 2000: 26-31) -lo que muestra que las instituciones aumentan la probabilidad de que opten por un curso de acción- sino también de ser víctimas de delitos (KARMEN, 2000: 31-34) - lo que muestra que las mismas instituciones aumentan la probabilidad de que les acaezca un estado de cosas-.

${ }^{28}$ Una peculiaridad adicional del orden internacional radica en que los encargados de aplicar este orden institucional internacional son los mismos Estados, bien individualmente o de modo colectivo. Remarcando este extremo con relación a las normas internacionales de derechos humanos, BEITZ señala que estas son estatistas en dos sentidos: «...(they) apply in the first instance to states, and they rely on states, individuals and in collaboration, as their principal guarantors...» (BEITZ, 2009: 128). 
institucionales que se aplican coercitivamente. El hecho de que los individuos habiten en algún Estado y que no quede ningún territorio libre del control estatal a donde quien no acepta esta forma de organización política pude emigrar, son algunas de las consecuencias que el orden internacional de modo coercitivo impone sobre todos los individuos que habitan el planeta ${ }^{29}$.

En síntesis, las instituciones estatales e internacionales no sólo se diferencian porque estas últimas se aplican a los Estados. Aunque ambas se aplican a los individuos, los coaccionan a soportar estados de cosas o adoptar cursos de acción diferentes. El esquema internacional les coerciona a vivir en algún Estado, aunque no les coerciona a vivir en el Estado particular en que lo hacen. Aumenta la probabilidad de que un estado de cosas acaezca, a saber, el de que habiten algún esquema estatal e incide sobre la probabilidad de que adopten un curso de acción, a saber, el que no puedan optar por vivir en un territorio sin Estado. El esquema de instituciones domésticas, en cambio, los coerciona a vivir en el particular conjunto de instituciones domésticas que los establecen como destinatarios. Estas, como hemos visto, aumentan la probabilidad de que a quienes se aplican les acaezcan ciertos estados de cosas — tales como los referidos al nivel de sus expectativas vitales, recursos y derechos- o adopten ciertos cursos de acción - tales como seguir una carrera u otra, o en el caso extremo delinquir o no hacerlo-.

El esquema de instituciones internacionales no afecta a los individuos de modo directo —-más allá del hecho señalado de imponerles vivir en algún Estado- fijando sus expectativas vitales, recursos y derechos ${ }^{30}$. El esquema de instituciones internacionales puede aumentar la probabilidad de que los Estados se encuentren organizados de cierto modo - por ejemplo, tengan gobiernos estables y legítimos o posean dictaduras inestables- pero aun con esta probabilidad en pié lo que aumenta la probabilidad de que un ciudadano en particular reciba ciertos recursos, derechos y oportunidades, que aumentan la probabilidad que se encuentre en cierto estado de cosas o adopte algún curso de acción, es el modo en que el Estado efectivamente se organiza ${ }^{31}$.

${ }^{29}$ En este punto discrepo con PETTIT quien señala que este hecho no puede considerarse que sea uno impuesto intencionalmente o cuasi-intencionalmente por un agente. PETTiT señala que «...It is a brute fact or historical necessity —an obstacle created by nature — that there is no State-less territory available...» (РЕTTTT, 2009a: 52 y 2010). PETTIT considera que este hecho no es «...in itself the effect of dominating interference by the local state» y de allí concluye que es un hecho bruto. Aunque considero que lo primero es cierto, pienso que la conclusión no se sigue. Esto toda vez que aun es posible, como sostengo, que se trate de un efecto producido por la «interferencia dominante» o la aplicación coercitiva de un esquema institucional distinto al estatal, esto es, el esquema de instituciones internacionales. Si existiese otro orden internacional — distinto al construido por la paz de Westfalia - seguramente el hecho de que todo el territorio se encuentre repartido entre Estados internamente supremos y externamente independientes, no sería el caso. Este estado de cosas no es un hecho bruto, sino uno producido por ciertas instituciones.

30 J. COHEn y Ch. SAbel (2005) basándose en el trabajo de B. KingSbURY, N. KRisch y R. STEWART (2005) sobre la existencia de un derecho administrativo global, han propuesto la hipótesis de que el orden internacional está en proceso de cambio debido al surgimiento de instituciones globales con poder coercitivo directo sobre los individuos. «To a substancial and growing extent, then, rulemaking directly affecting the freedom of action of individuals, firms, and nation states (and the making of rules to regulate this rulemaking) is taking place...» (COHEN y SABEL, 2005: 765). Si el orden internacional está cambiando y ha surgido un nuevo poder político coercitivo internacional que se aplica directamente a los individuos, tal como sostienen COHEN y SABEL, es un problema que no puedo abordar aquí. No obstante es preciso destacar que si lo que señalan fuese cierto, todo el argumento ofrecido en el texto debería ser revisado.

31 Th. PogGe, por ejemplo, ha llamado la atención respecto de cómo dos normas del actual sistema internacional determinan — con independencia de cual sea la opinión o voluntad de los Estados- que 
Una analogía con las instituciones domésticas puede ayudar a comprender la idea. La configuración de las instituciones domésticas, he señalado, aumentan la probabilidad de que algunos individuos adopten un curso delictivo de acción. El individuo que delinque amenazando con un arma a otro es, podríamos decir, coaccionado a coaccionar. Quien es asaltado es coaccionado por el delincuente y las instituciones coaccionan al delincuente. Sin embargo, constatar esto no nos hace concluir que quien coacciona a la persona que sufre el asalto son las instituciones. Quien la coacciona, quien aumenta la probabilidad de que realice el curso de acción de entregarle objetos de su propiedad, es el asaltante.

Lo mismo puede señalarse con respecto a las instituciones internacionales. La configuración de estas instituciones aumenta la probabilidad de que los Estados adopten cierto diseño de sus instituciones domésticas. Si fruto de este diseño de las instituciones domésticas aumenta la probabilidad de que algunos ciudadanos, por ejemplo, vivan en una situación de pobreza o carezcan de oportunidades, la situación podría ser descripta como un caso de coacción para coaccionar. El orden internacional ha coaccionado a las instituciones domésticas - ha tenido efectos coactivos sobre éstaspara que estas coaccionen a los ciudadanos que en ellas habitan. Constatar esto, sin embargo, no debe hacernos concluir que quien coacciona a los ciudadanos que sufren la pobreza o la falta de oportunidades son las instituciones internacionales. Quien los coacciona, quien aumenta la probabilidad de que soporte este estado de cosas, es el modo en que se encuentran configuradas las instituciones estatales ${ }^{32}$.

\subsection{Coacción y exigencias de legitimidad}

El carácter coercitivo de las instituciones estatales e internacionales determina que sean moralmente problemáticas. Para ver por qué ello es así, el punto de partida no puede ser otro que una determinada concepción normativa de persona. Los requerimientos morales determinan el modo adecuado de tratar a un sujeto así concebido.

aquellos que detentan el gobierno gozaran de lo que llama el «borrowing privilege» y el «natural resources privilege». El primero establece que los Estados responden por los prestamos solicitados por sus gobernantes, con independencia de si el gobernante accedió al poder por medios legítimos o ilegítimos. El segundo, establece que los Estados quedan vinculados por los contratos que sobre sus recursos naturales hayan hecho sus gobernantes, nuevamente con independencia de su modo de acceso al poder. Una consecuencia de estas normas internacionales — que se produce con independencia de la voluntad de los Estados afectados- es que los países ricos en recursos sean propensos a tener golpes de Estado conducidos por personas ávidas de disponer del crédito y los recursos naturales del Estado. Las menores expectativas de estabilidad institucional de las que gozan estos Estados es determinada por el esquema de instituciones internacionales en el que se encuentran.

${ }^{32}$ Lo señalado permite comprender por qué los ciudadanos de Estados que son puestos en la misma situación por el orden internacional — por ejemplo, ambos son ricos en recursos naturales, sus gobiernos tienen el «borrowing privilege» y el «natural resources privilege»— tienen probabilidades de soportar estados de cosas o adoptar cursos de acción diferentes. Así, por ejemplo, Nigeria y Suecia son ricas en petróleo. En ambos casos el orden internacional ha aumentado la probabilidad de que cada Estado disponga de sus recursos, sus gobiernos tengan potestad para contraer deudas, y para disponer de los recursos naturales. No obstante, los ciudadanos de Nigeria y Suecia tienen probabilidades sumamente divergentes por lo que respecta a sus expectativas vitales, oportunidades y recursos. Nigerianos y suecos son coaccionados a soportar diferentes estados de cosas y adoptar diferentes cursos de acción. Este no podría ser el caso si quien los coaccionase fuese el orden internacional. 
La concepción normativa de persona que utilizaré es una que concibe a los individuos como agentes sensibles a razones ${ }^{33}$. Un agente sensible a razones posee dos aspiraciones: dirigir su vida a partir de sus propias consideraciones y que estas consideraciones sean genuinas razones ${ }^{34}$. Si su vida es dirigida a partir de las decisiones de otro o a partir de decisiones propias no fundadas en genuinas razones, sus intereses en tanto agente sensible a razones no han sido satisfechos.

El primero de estos intereses, el de dirigir su vida a partir de sus propias consideraciones es el que es amenazado por la existencia de un esquema institucional coercitivo con efectos profundos sobre los sujetos a quienes se aplica. Es esta amenaza planteada por la coercitividad a la que da respuesta la legitimidad política. Donde sea que tal amenaza se plantea, las exigencias de legitimidad política se aplican. Las exigencias morales de legitimidad son el correlato del carácter coercitivo de un esquema institucional.

La amenaza consiste en que los deberes y derechos, los cursos de acción, las oportunidades, los recursos, los fines e intereses, de los sujetos a quienes se impone el esquema coercitivo no sean determinados por ellos mismos. Este es el caso cuando existe un esquema institucional con profundos efectos sobre los sujetos a quienes se aplica, que a su vez es ajeno a los sujetos a quienes se impone. La amenaza a la que da lugar la existencia de un esquema coercitivo radica en la imposición de un esquema institucional ajeno. Si un esquema institucional que no es propio del sujeto incide sobre su vida, ésta no está siendo dirigida a partir de sus propias consideraciones.

El modo de conjurar esta amenaza consiste en hacer que allí donde sea necesaria la existencia de instituciones coercitivas, los sujetos a quienes se aplican sean sus autores $^{35}$. Si en tanto agente sensible a razones un individuo tiene interés en dirigir su vida a partir de sus propias consideraciones, en ser su autor, y si la incidencia del esquema institucional puede conspirar en contra de la satisfacción de aquel interés, la solución es hacer que todos aquellos a quienes se aplica sean autores del mismo. Si la amenaza que plantea la existencia de instituciones coercitivas sobre aquellos a quienes se aplican reside en que no puedan dirigir su vida a partir de sus propias consideraciones, la solución radica en hacer que el esquema de instituciones estatales sea propio de aquellos a quienes se aplica. La solución radica en hacerlos sus autores ${ }^{36}$.

33 Esta es la idea básica del contractualismo scaloniano (SCANLON, 1998).

34 Un modo de justificar esta concepción normativa de persona es sostener que lo más característico que posee el ser humano - y también lo más valioso- es su capacidad para evaluar y sopesar razones y para conducir su propia vida de acuerdo con ellas. Lo valioso de esta capacidad determina que tanto el interés en dirigir su vida a partir de sus consideraciones como el interés en que estas sean genuinas razones, sean moralmente relevantes para decidir qué nos debemos en nuestro trato mutuo.

No obstante, no creo que esta sea la única justificación posible. Otras justificaciones, sin tanto peso metafísico pienso que también se encuentran disponibles.

${ }^{35}$ Si la existencia de instituciones coercitivas no es necesaria, entonces las mismas no están justificadas y el deber moral que engendra su existencia es el de trabajar para su desaparición. Sólo en el seno de instituciones coercitivas que se encuentran moralmente justificadas aparecen las exigencias de legitimidad política o autoría.

36 Dado que uno de los objetivos del presente trabajo es determinar qué relación existe entre el auto-gobierno y la democracia, he soslayado el problema de determinar qué grupos poseen derecho a auto-gobernarse. Creo que esta pregunta no se vincula tanto al problema de cuando un Estado es legítimo o se auto-gobierna sino al problema de cuando la existencia de un Estado se encuentra moralmente justificada. El grupo que reclama un derecho a secesionar y a auto-gobernarse afirma que la existencia del Estado del cual forma parte 
No se trata de la exigencia de que el esquema institucional sea justo o correcto, sino de que sea propio de los sujetos a quienes se aplica. La exigencia moral que engendra la aplicación de las instituciones coercitivas es la de legitimidad política. Justicia y autoría —o legitimidad política - son dos cosas diferentes. Un conjunto de sujetos puede ser autor de un esquema institucional — puede existir legitimidad política y estarse auto-gobernando- aunque el esquema institucional sea injusto o incorrecto. Así como una decisión individual puede ser propia y no obstante equivocada, un esquema institucional puede ser propio de los sujetos a quienes se aplica, ser legítimo, y no obstante ser injusto o incorrecto. Lo contrario implicaría afirmar que cuando una decisión o esquema institucional es propia de un individuo, entonces indefectiblemente es justa o correcta, lo cual no es el caso. Nuestros errores son nuestros, pero lo mismo siguen siendo errores ${ }^{37}$.

El hecho de que tanto las instituciones domesticas como las internacionales posean carácter coercitivo genera exigencias de legitimidad política, auto-gobierno o autoría, en ambos niveles. No obstante, tales exigencias no son idénticas porque los sujetos sobre quienes dichos esquemas se aplican son diferentes. Mientras que en el caso de los esquemas institucionales estatales, son los individuos considerados por separado los que deben ser sus autores, en el caso del esquema institucional internacional los autores deben ser los Estados legítimos, o los individuos políticamente organizados. El orden internacional es legítimo si los Estados son sus autores y si garantiza que los ciudadanos sean los autores de las instituciones estatales en las que habitan.

¿Qué condiciones deben satisfacerse para que las instituciones coercitivas sean de autoría de aquellos a quienes se aplican? La autoría o legitimidad no se encuentra vinculada a lo que los individuos hacen respecto al esquema institucional — participando, consintiendo, etc.- sino a lo que las instituciones hacen respecto de los sujetos a quienes se aplican. Los sujetos a quienes se aplican las instituciones no son autores del diseño institucional porque lo configuren a través de su participación efectiva sino que es el diseño institucional el que los configura como autores. Si un esquema institucional satisface los intereses que los sujetos a quienes se aplica poseen en tanto autores entonces los transforma en tales. He denominado a esta concepción la «legitimidad como autoría» ${ }^{38}$.

no está moralmente justificada. Este es un problema sumamente interesante y ha generado un debate notablemente activo, sin embargo abordarlo excede los límites del presente trabajo. Agradezco a un árbitro anónimo por haberme hecho notar la necesidad de aclarar este punto.

37 BLAKE ha sugerido un argumento de corte rawlsiano que tiene por objeto mostrar que lo que justifica utilizar el recurso de la posición original para evaluar el diseño de ciertas instituciones reside en el hecho de que se trata de instituciones coercitivas. Su conclusión es que los principios de justicia — que se obtienen a partir de la posición original - solo son aptos para evaluar instituciones que se aplican de modo coercitivo. Señala al respecto: «... The criteria for membership within the group of people entitled to justificación through principles de liberal justice, on my account, is membership as citizen within the territorial state. This first stage determines the class of people to whom justification is owed; the fact of coercion is what mandates the provision of such justification...» (BLAKE, 2002: 289). Con independencia de si se trata de una buena reconstrucción del pensamiento de RAWLS, por las razones brindadas en el texto considero que esta posición es inadecuada. La exigencia moral que engendra la existencia de un esquema institucional coercitivo, es la de que sea propio de los sujetos a quienes se aplica, no la de que sea justo o correcto. Las exigencias engendradas por la coerción son de legitimidad y auto-gobierno, no de justicia.

38 La concepción completa de la «legitimidad como autoría», ha sido presentada en otro trabajo al que sigo. Aquí sólo puedo fundarme en ella sin ofrecer argumentos a su favor (SELEME, 2010). 
La estructura de la concepción de «legitimidad como autoría» es la siguiente. En primer lugar se determinan, a partir de una concepción normativa de sujeto, cuáles son los intereses que éstos poseen, en tanto autores, con relación a las instituciones que se les aplican coercitivamente. En segundo lugar, establece qué rasgos debe poseer un esquema institucional para satisfacer los intereses que los sujetos tienen en tanto autores y para configurarlos de este modo como autores del mismo.

Dado que las instituciones estatales se aplican coercitivamente de modo directo sobre los individuos - determinando sus cursos de acción, expectativas de vida, gustos, talentos y preferencias - es necesario identificar cuáles son los intereses de autoría que poseen los individuos considerados por separado. Si las instituciones estatales satisfacen estos intereses, entonces ubican a los individuos en el rol de autores y son legítimas. Adicionalmente, dado que las instituciones internacionales imponen coercitivamente el sistema de Estados sobre los individuos, es necesario identificar el modo en que las instituciones internacionales pueden ayudar a satisfacer los intereses de autoría que poseen los individuos con respecto a las instituciones estatales. Por último, dado que las instituciones internacionales se aplican coercitivamente a los Estados, es necesario identificar cuáles son los intereses de autoría que poseen los Estados, o los individuos organizados políticamente. Si las instituciones internacionales garantizan que los Estados satisfagan los intereses de autoría individual y ellas mismas satisfacen los intereses de autoría colectiva - esto es los intereses que tienen como miembros de una comunidad política estatal— entonces son legítimas.

En lo que sigue analizaré sucesivamente en qué consisten estos diferentes intereses de autoría que las instituciones estatales e internacionales deben satisfacer para ser legítimas. El objetivo es mostrar que la función de los derechos humanos es garantizar que los intereses de autoría de los individuos sean satisfechos por las instituciones estatales. La concepción de los derechos humanos que voy a presentar afirma que éstos son condiciones necesarias y suficientes de legitimidad política estatal.

\subsection{Legitimidad de las instituciones estatales: los intereses de autoría de los ciudadanos}

El principal interés que poseen los ciudadanos, en tanto autores, es el de participar en el diseño y configuración de las instituciones estatales. Si las instituciones hacen posible tal participación, entonces ubican a los ciudadanos en el rol de autores, son de autoría de los ciudadanos, esto es, son legítimas.

La participación política posee dos manifestaciones. La primera, se refiere a tomar parte en la toma de decisiones colectivas. La segunda, se refiere a la aceptación de las decisiones colectivas adoptadas. De modo que existen dos modos en que un esquema institucional puede no tratar como autores, no satisfacer los intereses que tienen como participantes, los ciudadanos. Por impedirles que sus opiniones u intereses cuenten a la hora de tomar decisiones colectivas, o por tratarlos como meros súbditos, receptores de órdenes, de quienes no se pretende aceptación sino sólo obediencia.

Para identificar los intereses que los ciudadanos tienen —en tanto autores- en relación con el diseño institucional un buen punto de partida es la determinación de 
los intereses ciudadanos realizada por Ch. BEITZ (1990). Dado que BEITZ reconoce que los ciudadanos ocupan un rol dual de autores y de sujetos de las instituciones, de lo que se trata es de distinguir entre los intereses que los ciudadanos tienen, cuáles se encuentran vinculados a su calidad de autores, esto es, a su rol de participante en los dos sentidos señalados ${ }^{39}$.

BEITZ identifica tres grupos de intereses vinculados con la ciudadanía: el interés en el reconocimiento, en el modo de tratamiento ${ }^{40} \mathrm{y}$ en la responsabilidad deliberativa. En lo que sigue me detendré a mostrar qué porción de estos intereses se encuentra vinculada a los dos aspectos antes señalados de la participación, esto es, cuáles de estos intereses son intereses que los ciudadanos tienen como autores.

El interés en el reconocimiento se encuentra vinculado al acceso a los roles públicos y a la participación en los procedimientos decisorios. Este interés se refiere a los efectos que tiene sobre la identidad pública el lugar que el procedimiento político de toma de decisión colectiva y la estructura de roles públicos asigna a los individuos. Se trata de intereses que los ciudadanos tienen en su calidad de autores, puesto que se encuentran vinculados a su calidad de participantes. Cuando las instituciones están diseñadas de tal modo que una persona es excluida enteramente del acceso a cualquier rol público o cuando los roles en los procedimientos decisorios reflejan la creencia social en la inferioridad de un grupo, no es satisfecho el interés en el reconocimiento que todos los ciudadanos tienen como autores de las instituciones que se les aplican. Si una persona o grupo de individuos es excluido del acceso a los roles públicos, y tal cosa se encuentra fundada en la creencia social de su inferioridad, entonces el esquema institucional no trata a los excluidos como autores, como participantes ${ }^{41}$.

El segundo de estos intereses, el referido al modo de tratamiento, se encuentra vinculado al tipo de participación que se realiza a través de la aceptación del esquema institucional por parte de la ciudadanía. El esquema debe estar diseñado de modo tal que posibilite el involucramiento de su voluntad. Lo que se opone a un esquema que hace posible la aceptación por parte de sus ciudadanos, es uno que solo descansa en el uso de la fuerza. Un esquema donde una parte de la población fuese esclava y estuviese obligada a trabajar a favor de otros, o no tuviese garantizado los medios materiales de subsistencia, o no tuviese protección en contra del maltrato o el homicidio, o no pudiese profesar cierta religión o pensamiento, o no tuviesen garantías en contra del trato arbitrario por parte de las autoridades, sería uno que solo descansaría en el uso de la fuerza. Las instituciones no se mantendrían en vigor por la aceptación de los ciudadanos — que de este modo serían participantes y autores— sino meramente por

39 Esta dualidad de roles es puesta de manifiesto por HоBвES quien, sin embargo, pone el énfasis en el rol que los ciudadanos tienen como «sujetos» o súbditos y no como «autores» de las decisiones colectivas. El único acto de «autoría» o de participación en HOBBES sería el de autorizar la formación de un gobierno donde su ulterior participación no tiene cabida. BEITZ ha llamado la atención sobre este rol dual y ha ofrecido una teoría que pretende explicitar los términos en que debería ser tratado un ciudadano como igual, dado los intereses fundamentales vinculados a su rol de «autor» y «sujeto» de la política (BEITZ, 1990).

40 BEITZ lo denomina «interés en el tratamiento equitativo».

${ }^{41}$ BEITZ señala que un procedimiento decisorio con estas características «...establish or reinforce the perception that some people's interests deserve less respect or concern than those of others simple in virtue of their membership in one rather than another social or ascriptive group...» (BEITZ, 1990: 110). Para que esto se dé, sin embargo, no basta que algunas personas sean excluidas. Es necesario, además, que tal exclusión se deba a la creencia de que poseen menos valor. 
el uso de la fuerza de sus gobernantes. Sin estos derechos la idea misma de sistema político — como sistema de cooperación social de cuyas decisiones son autores los ciudadanos- carece de sentido ${ }^{42}$.

En consecuencia, para satisfacer los intereses que los ciudadanos tienen en tanto autores, para posibilitarles participar como aceptantes, el esquema institucional tiene que garantizar ciertos derechos. El derecho a la vida, a los medios de subsistencia y a la integridad personal, la libertad de ocupación forzosa y de conciencia, la igualdad de trato por las autoridades, etc. Si estos derechos no están satisfechos, los ciudadanos no son tratados como autores y el esquema institucional es ilegitimo ${ }^{43}$. Estos derechos, y el de que ningún grupo puede ser excluido del acceso a los roles públicos en base a consideraciones fundadas en su inferioridad, son condiciones necesarias que cualquier esquema institucional debe satisfacer para ser legitimo.

Finalmente, el interés en la responsabilidad deliberativa hace referencia, al igual que el primero, a la posibilidad de participar en el proceso de toma de decisiones colectivas. Se trata del interés en que la resolución de los asuntos políticos pueda hacerse en base a una deliberación pública suficientemente informada, donde las opiniones o razones de los ciudadanos puedan ser consideradas y evaluadas responsablemente (BEITZ, 1990: 113-117). Este tercer interés es satisfecho cuando las instituciones son sensibles a las opiniones e intereses que sobre los asuntos públicos tienen los ciudadanos. Un sistema de toma de decisiones colectivas que impide que sean consideradas las opiniones de determinado grupo no satisface este interés en la responsabilidad deliberativa.

Un sujeto posee dos tipos de razones relevantes a nivel político. Unas, se refieren al contenido que deberían tener las decisiones colectivas. Por ejemplo, si se debe decidir colectivamente sobre la existencia de una opción pública para el seguro de salud, algunos tendrán razones a favor y otros en contra de tal propuesta. La satisfacción del interés en la responsabilidad deliberativa exige que el procedimiento de toma de decisiones colectivas no impida que las opiniones de todos sean consideradas. Otras, se refieren al procedimiento para adoptar decisiones colectivas. El objeto de estas razones u opiniones individuales no es el contenido de las decisiones colectivas — si debe existir una opción publica en el sistema de salud — sino los criterios que sirven para evaluar el

${ }^{42}$ Considero que este es el argumento que justifica los derechos humanos en la concepción política rawlsiana. Señala RAWLS: «... What have come to be called human rights are recognized as neccesary conditions of any system of social cooperation. When they are regularly violated, we have command by force, a slave system, and no cooperation of any kind» (RAWLS, 1999: 68).

${ }_{43}$ En este punto he seguido la exposición que RAWLS realiza con relación a las condiciones con un esquema institucional debe satisfacer para ser un esquema de cooperación. RAWLS contrapone dicho esquema con lo que denomina una «slave society». En la terminología que he utilizado, tal sociedad seria una que no permitiría que sus ciudadanos fuesen aceptantes, esto es, descansaría meramente en el uso de la fuerza. Señala RAWLS: «...A slave society lacks a decent system of law, as its salve evonomy is driven by a scheme of political and social cooperación...» (RAWLS, 1999: 65). A diferencia de RAWLS, sin embargo, no considero que los derechos humanos puedan reducirse a aquellos que garantizan que los ciudadanos puedan ser aceptantes. Los derechos humanos garantizan la legitimidad de un esquema institucional —evitando que sea una «slave society»— pero para hacerlo deben ser mas extensos que lo que Rawls piensa. Básicamente, deben incluir los derechos que permiten satisfacer los otros dos intereses vinculados con la autoria. La idea misma de una «sociedad de esclavos» encaja con la concepción de la «legitimidad como autoría» que he presentado. Tal sociedad es una donde sus ciudadanos no tienen poder para dirigir sus propias vidas, estos no son autores. 
procedimiento decisorio — si la decisión debe tomarse por votación o no, por mayoría simple o calificada, etc.- -

Ahora bien, si los ciudadanos pueden poseer razones de los dos tipos señalados existen dos modos en que el interés deliberativo puede ser vulnerado: por utilizar un procedimiento de toma de decisión colectiva que impide que las consideraciones de algunos individuos respecto al contenido de la decisión colectiva sean escuchadas, o por utilizar un procedimiento que - aunque permite que las opiniones de todos sean escuchadas - se encuentre fundado en consideraciones que no puedan ser vistas como razones por todos los individuos en cuestión. En ambos casos el sistema institucional impide que las razones u opiniones de los ciudadanos cuenten y, por tanto, no satisface su interés deliberativo.

En otras palabras, existen dos niveles en que el esquema institucional satisface el interés en la responsabilidad deliberativa de los ciudadanos. El primero se refiere al procedimiento de toma de decisiones colectivas que emplea el esquema institucional estatal. Este debe permitir que sean consideradas toda opinión acerca del contenido de las decisiones públicas. Si el procedimiento impide que algunas opiniones sobre la marcha de los asuntos públicos sean escuchadas, el interés en la responsabilidad deliberativa no está siendo satisfecho y el esquema institucional no trata a los ciudadanos como autores. A la hora de determinar el contenido de una decisión colectiva el ciudadano es tratado como autor por el esquema institucional si no existen impedimentos para su participación, esto es para que sus opiniones e intereses cuenten en el procedimiento de toma de decisiones.

El segundo, más profundo, se refiere a los criterios para diseñar y evaluar dicho procedimiento decisorio. Con relación a las opiniones que los ciudadanos tienen respecto al procedimiento mismo de toma de decisiones colectivas, en cambio, el interés en la responsabilidad deliberativa no puede ser satisfecho por la existencia de un procedimiento que permita que las opiniones de todos los ciudadanos cuenten. Debe ser satisfecho en otro nivel. La razón de ello radica en que las opiniones de los ciudadanos se refieren al procedimiento decisorio mismo. El mecanismo por el cual un diseño institucional es sensible a las opiniones que los ciudadanos poseen respecto al procedimiento de toma de decisión colectiva no puede ser la existencia de un procedimiento de toma de decisión colectiva en el cual nadie se encuentre impedido de expresar su opinión. Esto presupone ya haber adoptado un procedimiento de toma de decisiones colectivas sobre el cual los ciudadanos tienen diversas opiniones.

Del mismo modo, a la hora de determinar el procedimiento de decisión colectiva el ciudadano es tratado como autor si no existen impedimentos para su participación. Sin embargo, la participación de la que se trata aquí no puede consistir en tomar parte en un procedimiento de decisión colectiva puesto que de lo que se trata es justamente de establecer cuál es este procedimiento. La única participación que puede darse aquí es a nivel de razones. Un ciudadano ha participado en la adopción de un procedimiento de toma de decisiones colectivas, si las consideraciones que lo justifican son aquellas que él efectivamente puede juzgar como razones. En consecuencia, un ciudadano es tratado como autor en este segundo nivel si no se encuentra impedido de participar, esto es si las consideraciones que fundan el procedimiento de toma de decisiones colectivas pueden ser consideradas como razones por él. Un procedimiento de toma de decisiones colectivas permite satisfacer el interés en la responsabilidad deliberativa 
cuando se encuentra fundado en consideraciones que ningún ciudadano se encuentra impedido de ver como una razón ${ }^{44}$.

Lo que posibilita que un conjunto de individuos pueda juzgar en común a una determinada consideración como una razón es el hecho de que comparten una misma cultura política pública. Comparten una práctica argumentativa referida a la toma de decisiones colectivas y al diseño institucional, la cual descansa en ideas implícitamente compartidas por todos los participantes. Un procedimiento de toma de decisiones colectivas que se encuentra justificado a partir de estas ideas, es uno que puede ser visto como fundado en razones por cualquier ciudadano en tanto miembro de una misma cultura política, participante de una práctica argumentativa.

Cómo es obvio esto no equivale a señalar que el carácter de razón de una consideración le viene dado por una cultura, sino simplemente que el juicio sobre qué cuenta como una razón es relativo a un determinado ambiente cultural. Mientras más densa sea la cultura común, más posibilidades existirán de juzgar conjuntamente a determinadas consideraciones como razones. En consecuencia, el modo de satisfacer el interés en la responsabilidad deliberativa, esto es el modo en que un esquema institucional trata a sus ciudadanos como autores, dependerá de las características culturales que posea la sociedad en cuestión.

Introducir una precisión puede ser de utilidad para aclarar las ideas expresadas. Es necesario distinguir tres tipos de juicios. El primero, se refiere al procedimiento de toma de decisiones utilizado por un determinado grupo social. Si este procedimiento es sensible a las opiniones - vinculados con el contenido de las decisiones- de aquellos a quienes se van a aplicar las decisiones coercitivas adoptadas, entonces cuenta como un procedimiento de toma de decisiones colectivas. Este primer tipo de juicio no es sensible a la cultura ni a las opiniones que tienen los involucrados. Lo que se exige aquí, es que en alguna medida las opiniones o razones de todos los involucrados cuenten en el proceso de toma de decisión ${ }^{45}$. El segundo, se refiere a las razones que justifi-

44 Una consideración puede ser vista por un individuo como una razón cuando es posible vincularla a las creencias, compromisos, proyectos, valores, etc., que actualmente posee. Sólo en este caso, algo es una razón para él o una razón suya. La idea es semejante — aunque no idéntica- a la defendida por B. WILLIAMS (1981), por lo menos en una de sus interpretaciones. WILLIAMS sostiene que afirmar que un individuo tiene una razón implica afirmar que existe una «sound deliberative route» entre el conjunto motivacional S del individuo y la realización de determinado comportamiento. Análogamente en el texto sostengo que para que alguien pueda percibir a determinada consideración como una razón tiene que poderla vincular mediante un proceso de deliberación correcta con algún componente subjetivo — que no necesariamente debe tener carácter motivacional- (creencias, valores, deseos, disposiciones de evaluación, etc.) que actualmente posee. Lo señalado en el texto es compatible con admitir que los individuos pueden modificar sus razones a través de lo que SCANLON denomina la «modificación reflexiva». Sostener que algo es visto como una razón por un individuo cuando puede ser vinculada a algún elemento subjetivo del mismo, no equivale a adoptar una posición quietista en relación con las razones. Las consideraciones que reconocemos como razones pueden cambiar, pero sólo haciendo pie en algún componente subjetivo. El procedimiento a través del cual «...one decides what reasons one has, depend on the reactions that the person doing the deciding has or would have to the distinctions, examples, and analogies in question...» (SCANLON, 1998: 368). Un individuo se encuentra impedido de juzgar a una consideración como una razón cuando no es posible vincularla por deliberación con ningún componente subjetivo.

45 Esto permite excluir los sistemas que segregan a la hora de tomar decisiones colectivas a una porción de la población, aun si se encuentra fundado en una concepción de persona segregacionista que se encuentra implícita en la cultura política pública. Este es el único modo de satisfacer los intereses que los ciudadanos tienen como «autores». Considerar a estos sistemas como mecanismos de toma de decisión colectiva implicaría tener deferencia por las razones que poseen vinculadas a cómo debería configurarse el procedimiento de toma 
can adoptar una decisión política en lugar de otra. Este juicio, obviamente, es sensible a la cultura y a las opiniones que en relación con el contenido de la decisión tienen los involucrados. Finalmente, un tercer tipo de juicios se refiere a las consideraciones que justifican adoptar un procedimiento de decisión. Al igual que el anterior este juicio también es sensible a la cultura y a las opiniones de los ciudadanos. El procedimiento decisorio debe estar fundado en consideraciones que puedan ser vistas como razones por los involucrados ${ }^{46}$.

En síntesis, un esquema institucional estatal es legitimo - satisface los intereses que los ciudadanos tienen en tanto autores - cuando: a) el procedimiento de toma de decisiones colectivas es sensible a sus opiniones sobre la marcha de los asuntos públicos y las consideraciones que justifican adoptar este procedimiento puedan ser vistas como razones por los ciudadanos, dada la cultura política pública en la que habitan; b) les son reconocidos a los ciudadanos los derechos mínimos indispensables para que el esquema institucional no esté basado sólo en el uso de la fuerza sino que sea capaz de involucrar su voluntad y ganar su aceptación, y c) no están excluidos del acceso a cargos públicos debido a la creencia social sobre su falta de valor.

\subsection{Legitimidad de las instituciones internacionales}

El carácter coactivo de las instituciones internacionales engendra, de igual manera, la exigencia moral de satisfacer los intereses de autoría o de legitimidad política. No obstante, como he señalado, las instituciones internacionales presentan una peculiaridad que determina que las exigencias de legitimidad tengan rasgos distintivos. En primer lugar, dado que se aplican de modo coercitivo a los Estados, deben satisfacer los intereses que los Estados poseen en tanto autores del orden internacional. En segundo lugar, dado que las instituciones internacionales de modo coercitivo imponen sobre los individuos un sistema de Estados cuyas instituciones se les aplican coercitivamente, deben garantizar que los intereses que los ciudadanos poseen en tanto autores de los esquemas institucionales estatales sean satisfechos por los Estados.

de decisión pero implicaría no tener deferencia por las opiniones que tienen en relación al contenido de las decisiones políticas. El único modo de mostrar deferencia en relación con los dos niveles de razones que poseen es a través de un sistema de toma de decisiones colectivas que es a la vez sensible a las opiniones de todos respecto a la marcha de los asuntos públicos cuyo diseño está fundado en consideraciones que todos pueden ver como razones. Un sistema segregacionista satisface la segunda de estas exigencias pero no la primera.

46 Es necesario aquí realizar una aclaración para diferenciar mi posición de la defendida por algunos comunitaristas. Al igual que ellos considero que el juicio sobre qué cuenta para nosotros como una razón es sensible a nuestra cultura. Es sensible a quienes de hecho somos. En este sentido, y sólo en éste, es cierta la afirmación de WALZER: «...the question most likely to arise in the minds of the members of the political community is not, what would racional individuals choose under universalizing condictions of such-and-such a sort? But rather, what would individuals like us choose, who are situated as we are, who share a culture and are determined to go on sharing it? And this is a question that is readily transformed into, what choices have we already made in the course of our common life? What understandings do we (really) share?...» (WALZER, 1983: 5). Interpretada, por un lado, como referida a las condiciones del auto-gobierno - y no como referida a la justicia distributiva como el propio WALZER hace- $y$, por otro, como haciendo referencia a lo que determina que algo cuente como una razón moral para nosotros - y no a lo que constituye a una consideración en una razón moral — la afirmación de WALZER es correcta. En lo que se refiere a las condiciones del auto-gobierno y la legitimidad política, las preocupaciones de los comunitaristas referidas al carácter abstracto de los test liberales resultan fundadas. No resultan fundadas cuando se las extiende, como ellos hacen, a cuestiones de justicia. 


\subsubsection{Los derechos bumanos como correlato de la imposición coercitiva del sistema de Estados}

Que el sistema de instituciones internacionales deba reconocer y proteger los derechos humanos se relaciona con el segundo de los rasgos antes señalados. El hecho de que el esquema de instituciones internacionales de modo coercitivo establezca un sistema de Estados, que a su vez son esquemas que coercitivamente se aplican a quienes los habitan, determina que el carácter coercitivo de las instituciones estatales no sólo engendre exigencias morales respecto a cómo deben encontrarse diseñadas las instituciones estatales, sino también al modo en que dicho diseño debe ser garantizado por las instituciones internacionales. Las instituciones internacionales deben garantizar que los derechos asociados a los intereses de autoría de los ciudadanos se encuentren satisfechos por las instituciones estatales cuya existencia es coercitivamente establecida por el esquema internacional. Los derechos humanos son la herramienta a través de la cual el orden internacional vuelve efectiva esta garantía.

Que los derechos humanos sean la contrapartida de estos dos niveles de coacción que caracteriza al sistema de Estados creado por las instituciones internacionales - que imponga coercitivamente un esquema de instituciones estatales que a su vez son coercitivas- explica uno de sus rasgos más característicos. La satisfacción de los derechos humanos es una exigencia que en primer lugar compete a cada Estado respecto a sus propios ciudadanos y, en calidad de garante, a la comunidad internacional. Los derechos humanos son requerimientos morales cuyos destinatarios son los Estados, siendo la comunidad internacional un mero garante de segundo orden. La razón de esta peculiaridad es que los Estados deben satisfacer los requerimientos de legitimidad engendrados por la coacción que ejercen sobre sus ciudadanos y el esquema internacional que impone sobre los individuos un sistema de Estados debe garantizar - a través de la exigencia de los derechos humanos- que así lo hagan.

Tomando las conclusiones obtenidas en el apartado anterior, la idea sería la siguiente. El carácter coactivo de las instituciones estatales determina que las mismas deban satisfacer los intereses de autoría de los ciudadanos, lo cual alcanzan mediante la concesión de ciertos derechos a los ciudadanos. El esquema de instituciones internacionales, a su vez, establece al Estado como el modo que las organizaciones políticas paradigmáticamente deben adoptar. El carácter coactivo de las instituciones internacionales determina que las mismas deban garantizar que los esquemas estatales concedan a sus ciudadanos los derechos que les permiten satisfacer sus intereses de autoría. Los derechos humanos suministran esta garantía.

Si el esquema institucional estatal contiene los derechos requeridos para proteger los tres intereses de autoría de sus ciudadanos, entonces la exigencia moral que surge del carácter coactivo de las instituciones estatales se encuentra saldada y el esquema institucional estatal es legítimo. No obstante, como hemos señalado, todavía restan otras exigencias surgidas del carácter coactivo no ya del orden estatal sino del internacional. Para satisfacer los intereses de autoría de los individuos sobre quienes impone un sistema de Estados, el orden internacional debe exigir y garantizar que los Estados satisfagan las condiciones de legitimidad antes señaladas. Esto es, debe exigir 
que los Estados protejan los derechos que son condiciones necesarias y suficientes de legitimidad estatal, cuya protección implica que los intereses de autoría de los ciudadanos se encuentren satisfechos. Dicho de modo sintético, así como el hecho de que los esquemas estatales protejan ciertos derechos es condición de su legitimidad, que el orden internacional se presente como garante de estos derechos elevándolos al rango de derechos humanos es condición de la suya.

Sobre el orden internacional recae la exigencia moral —surgida de la imposición coercitiva del sistema de Estados- de garantizar que los Estados protejan los derechos civiles, sociales y políticos que permiten ubicarlos en el rol de autores. Sobre los Estados recae la exigencia moral —surgida de la imposición coercitiva de sus instituciones sobre los ciudadanos- de proteger estos derechos. Si un Estado no protege los derechos de sus ciudadanos no los trata como autores y es, por tanto, ilegítimo. Si el orden internacional no exige y es garante de esta protección - a través del instrumento de los derechos humanos- carece igualmente de legitimidad. En el primer caso las exigencias morales surgidas de la imposición coactiva de un particular esquema de instituciones estatales quedan insatisfechas. En el segundo, las que no son satisfechas son las exigencias morales surgidas de la imposición del sistema internacional de Estados por parte del orden internacional.

Estos dos niveles de responsabilidad — estatal e internacional— surgida de dos niveles de coacción, ha comenzado a ser reconocida de manera explícita en algunos instrumentos internacionales. De especial relevancia ha sido el reporte de la COMISIÓN INTERNACIONAL SOBRE INTERVENCIÓN Y SOBERANÍA DE LOS ESTADOS denominado The Responsibility to Protect (ICISS, 2001) ${ }^{47}$. Allí de modo expreso se señala que cada Estado tiene la responsabilidad de garantizar los derechos humanos de sus ciudadanos. Si es incapaz de hacerlo o si no tiene la voluntad política de hacerlo, entonces tal responsabilidad recae en la Comunidad Internacional.

\subsubsection{Los derechos de los Estados como correlato de la imposición coercitiva de un orden internacional}

Garantizar los derechos humanos, no obstante, no es la única exigencia que las instituciones internacionales deben satisfacer para ser legítimas. Que las instituciones internacionales se apliquen coercitivamente a los Estados engendra otras exigencias de legitimidad. Al igual que en el caso de las instituciones estatales, las instituciones internacionales deben satisfacer los intereses de autoría de los sujetos a quienes se aplican, esto es de los Estados legítimos o de los individuos considerados como cuerpo políticamente organizado ${ }^{48}$.

47 La comisión fue presentada por el gobierno de Canadá como respuesta a un requerimiento del secretario general Kofi Annan. El objetivo era ofrecer una solución al conflicto que existía entre el principio de soberanía nacional y el derecho de la ONU a intervenir donde se estuviesen violando derechos humanos. La comisión estuvo dirigida por Gareth Evans, quien se había desempeñado como ministro australiano de Asuntos Exteriores.

La existencia de dos niveles de responsabilidad también ha sido reconocida por la Asamblea de las Naciones Unidas. En el año 2005, los asistentes a la Sexagésima Asamblea General acordaron que existía una responsabilidad universal en la protección de los derechos humanos (ONU, 2005).

48 Esto se sigue de la tesitura liberal que no existen tales cosas como entidades colectivas supra-individuales. Lo que existen son individuos colectivamente organizados, por lo que los intereses relevantes siguen siendo los intereses individuales. 
Extrapolando los tres intereses de autoría —el interés en el reconocimiento, en el modo de tratamiento y el de la responsabilidad deliberativa - al ámbito internacional es posible establecer las condiciones que éste debe satisfacer para que su aplicación coactiva se encuentre justificada ${ }^{49}$. Por lo que respecta al interés en el reconocimiento es necesario que las instituciones internacionales se encuentren diseñadas de tal modo que ningún Estado legítimo esté excluido del acceso a ciertos roles públicos en las instituciones internacionales en base a una creencia en su inferioridad. Si un Estado es excluido de cierto rol en el esquema de instituciones internacionales, el esquema institucional internacional no lo trata como autor y es, por tanto, ilegítimo. El modo en que se encuentra organizado el Consejo de Seguridad de las Naciones Unidas —con miembros permanentes y rotativos- sufre de este defecto.

Por lo que respecta al modo de tratamiento, es necesario que las instituciones internacionales se encuentren diseñadas de tal modo que no sea irracional para los Estados, obedecerlas de modo voluntario. Esto es, deben posibilitar el involucramiento de la voluntad de los Estados ${ }^{50}$. El esquema de instituciones internacionales debe tener un diseño tal que su cumplimiento no sólo descanse en el uso de la fuerza. El modo de alcanzar tal extremo es garantizando que los Estados dispongan de los derechos y recursos necesarios para llevar adelante sus fines en tanto comunidades políticas legítimas. Un esquema de instituciones internacionales que no prohíbe la intervención de un Estado en los asuntos de otros - probado que este posee un orden institucional legítimo- o que no garantiza que cada Estado posea los recursos materiales necesarios mínimos para poseer un esquema institucional legítimo o no prohíbe la agresión bélica, sería ilegítimo.

Finalmente, quedan los rasgos que deben poseer las instituciones internacionales de modo de satisfacer el interés que los individuos —organizados políticamente en Estados - tienen en la responsabilidad deliberativa. A semejanza de lo que sucedía a nivel doméstico, los Estados tienen un interés en que la resolución de los asuntos colectivos pueda hacerse en base a una deliberación pública informada donde sus razones puedan ser consideradas y evaluadas. El modo de satisfacer este interés es a través de un sistema de toma de decisiones colectivas que no impida que las opiniones de algún Estado legítimo cuenten.

Puesto que los individuos políticamente organizados también poseen opiniones de los dos tipos que hemos señalado con anterioridad —opiniones respecto del contenido de las decisiones colectivas y opiniones respecto al procedimiento para adoptar decisiones colectivas- es necesario que ambas sean consideradas. Si el procedimiento de toma de decisiones a nivel internacional impide que sean consideradas las opiniones de algún Estado legítimo respecto del contenido de las decisiones colectivas, o si el procedimiento mismo se encuentra fundado en consideraciones que no pueden ser vistas como razones por todos los Estados legítimos, el sistema institucional impide

49 Lo señalado aquí se funda en una concepción normativa de Estado legítimo o de comunidad política legítima elaborada en el apartado anterior. A partir de esta concepción es que se identifican los intereses de los Estados legítimos, o de los individuos políticamente organizados.

50 No se trata de que los Estados posean una voluntad en el mismo sentido que los individuos que los componen. Se trata, simplemente, de que los individuos políticamente organizados poseen intereses que no poseen hasta que esto sucede y que dichos intereses son relevantes a la hora de motivarlos a actuar. 
que las razones u opiniones de los Estados cuenten y, por tanto, no satisface su interés deliberativo. Abordaré el problema de cuál es el procedimiento legítimo de toma de decisiones a nivel internacional en la sección siguiente al tratar el asunto de la Democracia Internacional.

\section{DERECHO HUMANO A LA DEMOCRACIA Y DERECHO A LA DEMOCRACIA INTERNACIONAL}

Habiendo establecido el doble conjunto de exigencias de legitimidad que pesan sobre el esquema internacional - las corporizadas en los derechos humanos y en los derechos de los Estados_- es posible finalmente abordar la cuestión de si entre estas se encuentra la democracia. Específicamente, es posible determinar si existe un Derecho Humano a la Democracia, del cual deban ser garantes las instituciones internacionales, o un derecho a la Democracia Internacional.

\subsection{La democracia como derecho humano}

Dado que la democracia es un procedimiento para adoptar decisiones colectivas, determinar si se trata o no de un derecho humano equivale a establecer si la adopción de dicho procedimiento a nivel estatal es condición necesaria para que algunos de los intereses de autoría se encuentren satisfechos. Si el interés en el reconocimiento, en el modo de tratamiento o en la responsabilidad deliberativa no es satisfecho a menos que el mecanismo de toma de decisión sea democrático, entonces la democracia es una condición que todo sistema estatal debe satisfacer para tratar a sus ciudadanos como autores, de la cual el sistema internacional debe ser garante. En este supuesto, existiría un Derecho Humano a la Democracia. Si por el contrario, un Estado puede satisfacer los intereses de autoría de sus ciudadanos sin ser democrático, entonces no existiría tal cosa como un Derecho Humano a la Democracia.

Estrictamente hablando, si se interpreta a la democracia como un procedimiento de toma de decisiones colectivas, el único interés de autoría involucrado debería ser el de la responsabilidad deliberativa. Si es posible que algunos individuos se encuentren imposibilitados de ver a las consideraciones que justifican el procedimiento democrático como razones, entonces su interés en la responsabilidad deliberativa no estaría satisfecho. En este supuesto, aunque el procedimiento sería sensible a sus razones con respecto al contenido de las decisiones colectivas, no sería sensible a sus razones con respecto a la configuración que debería tener el propio procedimiento.

Como hemos señalado, lo que posibilita que un conjunto de individuos pueda juzgar en común a una determinada consideración como una razón es el hecho de que compartan ciertas ideas implícitas en su cultura política pública. Sólo si el procedimiento de toma de decisiones colectivas se encuentra justificado a partir de estas ideas, entonces puede ser visto como fundado en razones por cualquier ciudadano a quien se aplica. Para determinar, entonces, si el procedimiento democrático satisface el interés en la responsabilidad deliberativa es necesario establecer si las ideas que lo justifican se encuentran implícitas en cualquier cultura política. 
Identificar las razones morales que justifican la regla democrática de toma de decisiones es relativamente sencillo. Lo que subyace a dicho procedimiento es la idea de ciudadanía libre e igual. Las decisiones deben ser adoptadas por un procedimiento en el que todas las opiniones cuentan y se sigue la opinión de la mayoría, porque todos los ciudadanos tienen un igual derecho a la participación. Ahora bien, si esta es la razón que justifica la democracia, parece que existen buenos motivos para dudar de que su implementación satisfaga el interés en la responsabilidad deliberativa.

En aquellas sociedades donde la cultura política no contiene implícita la idea de ciudadanía libre e igual, adoptar un procedimiento democrático no permitiría tratar a sus ciudadanos como autores. En estas sociedades debería optarse por un procedimiento que, en primer lugar, considerase las opiniones de todos sobre el contenido de las decisiones colectivas aun si no las considerase por igual — dado que no es democrático- $-\mathrm{y}$, en segundo lugar, se encontrase justificado en consideraciones que, dada la cultura política, puedan ser vistas por todos como razones.

La sociedad islámica imaginada por RAWLS en The Law of Peoples, con su procedimiento decisorio de consulta jerárquica, es un ejemplo de lo recién señalado (RAWLS, 1999: 64). La idea de persona implícita en la cultura política de esta sociedad es de índole comunitarista. Los ciudadanos se perciben como vinculados a sus grupos de pertenencia con lazos constitutivos, y no consideran que pueda haber discrepancia entre sus intereses y los de su comunidad de pertenencia. En este tipo de sociedad un mecanismo de consulta, donde las opiniones de los miembros de cada comunidad son consideradas por sus jefes, y las de estos por sus superiores en la escala jerárquica, sería el adecuado. No todas las opiniones sobre los asuntos públicos serian consideradas por igual, pero en alguna medida todas lo serian, y todos podrían ver como razones a las consideraciones que justifican dicho mecanismo de decisión. Adoptar en esta sociedad un procedimiento democrático impedirá satisfacer el interés en la responsabilidad deliberativa de sus ciudadanos y, al hacerlo, no los trataría como autores. En una cultura política semejante el único Estado que puede satisfacer los intereses de autoría de sus ciudadanos es uno que no sea democrático ${ }^{51}$.

Lo señalado, sin embargo, no basta para mostrar que la democracia no es una condición necesaria de legitimidad política que deba ser protegida internacionalmente a través del recurso de los derechos humanos. Si este procedimiento de toma de decisiones colectivas fuese el único compatible con la satisfacción de los otros intereses de autoría - el interés en el reconocimiento y el modo de tratamiento- entonces sólo los sistemas democráticos serían legítimos. Sólo en aquellas culturas que tienen implícita la idea de ciudadanía libre e igual podrían existir sistemas legítimos, porque sólo en ellas podrán estar satisfechos los tres intereses de autoría.

El argumento a favor de esta posición hace hincapié en el carácter igualitario que posee el procedimiento de toma de decisiones democrático. Sólo un sistema que con-

51 Lo señalado en el texto debe distinguirse del argumento que señala que promover el surgimiento de la democracia en sociedades que no tienen una cultura política firme puede conducir a resultados indeseables. J. KIRKPATRICK llama la atención acerca de lo riesgoso de promover la democracia en estas sociedades: «Hurried efforts to force complex and unfamiliar political practices on societies lacking the requisite political culture, tradition, and social structures not only fail to produce desired outcomes; if they are undertaken at a time when the traditional regime is under attack, they actually facilitate the job of the insurgents» (KIRKPATRICK, 1979: 37 ). 
fiere derechos iguales a todos los ciudadanos - incluidos los derechos para tomar parte en la toma de decisiones colectivas - los trata a todos por igual como autores. Un sistema en el que no todos pueden participar en pie de igualdad en la toma de decisiones - porque algunas decisiones son dejadas en manos de quienes se encuentran arriba en la escala jerárquica- no satisface, continúa el argumento, el interés en el reconocimiento. El que los ciudadanos sean reconocidos como autores por el sistema requiere que exista un derecho igual a la participación política, y esta igualdad sólo se logra si se adopta el proceso de toma de decisión democrática. Lo mismo se aplica al interés en el modo de tratamiento. Un sistema en el que no hay igualdad de derechos, señala el argumento, es incapaz de involucrar la voluntad de los ciudadanos y sólo puede ser mantenido por la fuerza. Finalmente, un sistema que no es igualmente sensible a las opiniones de todos sobre el contenido de las decisiones colectivas, porque confiere mayor peso a las opiniones de algunos — por ejemplo, quienes se encuentran en estratos jerárquicos superiores - no respeta uno de los intereses vinculados a la responsabilidad deliberativa. Si el proceso de toma de decisiones no es democrático, aun si esto obedece a que es sensible a las razones que los ciudadanos tienen acerca de cómo deben adoptarse las decisiones colectivas, no será igualmente sensible a las opiniones que los ciudadanos tienen sobre el contenido de dichas decisiones.

La estructura de estos argumentos es semejante. Parten, en primer lugar, de la premisa que las exigencias de autoría —o de legitimidad— son de índole igualitaria. A continuación señalan que sólo un sistema que confiere derechos iguales a todos sus ciudadanos es capaz de satisfacer los intereses de autoría, o las exigencias igualitarias de legitimidad. Finalmente, ponen de manifiesto el carácter igualitario del procedimiento de toma de decisiones democrático. Sólo con este procedimiento se garantiza que todos tengan un derecho igual a la participación política. De allí concluyen que el procedimiento democrático es el único que permite satisfacer los intereses de autoría de los ciudadanos.

Lo malo de estos argumentos es que parten de una premisa falsa. Concebir a la legitimidad como autoría permite comprender una característica de las exigencias de legitimidad que un esquema institucional debe satisfacer. Estos requerimientos, contrario a lo que es comúnmente sostenido, no son de índole igualitaria —como afirma la primera premisa - sino suficientista. La razón de esto reside en que es perfectamente posible ser co-autor de un emprendimiento colectivo sin haber contribuido por igual a su realización. Por ejemplo, ser co-autor de un libro no requiere que todas las personas involucradas escriban un número igual de páginas, sino más bien haber escrito un número suficiente. Aun si el grado de participación de todos los involucrados en una empresa colectiva no es igual, si cierto umbral de suficiencia es satisfecho, todos son igualmente autores. Esto permite percibir la naturaleza suficientista que poseen los intereses de autoría.

Lo dicho puede ser aplicado a las exigencias de legitimidad. En tanto co-autores, los ciudadanos no están interesados en una igual participación sino simplemente en una participación que satisfaga los niveles de suficiencia requeridos. Los intereses de autoría que los ciudadanos tienen con respecto al esquema institucional no son de índole igualitaria sino suficientista. Ellos están interesados en que el papel que cumplen en el esquema institucional no los excluya del proceso de toma de decisiones colectivas en base a una creencia en su inferioridad. Sin embargo, esto es compatible con no tener 
igual acceso a los roles públicos o no tener una posición en pié de igualdad en el proceso de toma de decisiones colectivas ${ }^{52}$. Están también interesados en tener los derechos mínimos requeridos para que el esquema institucional no esté fundado en el uso de la fuerza. No obstante, esto es compatible con no tener derechos iguales ${ }^{53}$. Finalmente, tienen interés en que sus opiniones e intereses no sean excluidos del proceso de toma de decisión colectiva. Sin embargo, esto es consistente con el hecho de que estas opiniones e intereses no reciban igual consideración en dicho proceso ${ }^{54}$.

En base a lo señalado es posible advertir porqué no existe un Derecho Humano a la Democracia. La adopción de un procedimiento democrático de toma de decisiones no es condición necesaria para que los intereses de autoría se encuentren satisfechos. Es posible que existan Estados legítimos que no son democráticos y, por ende, la carencia de democracia no es una amenaza en contra de la cual el sistema internacional deba brindar protección a través de los derechos humanos. Todos los seres humanos tienen un interés genérico en que el procedimiento de toma de decisiones sea sensible a sus opiniones - de los dos tipos que hemos especificado- pero el procedimiento de decisión que un Estado debe adoptar, y los derechos asociados al mismo que debe garantizar, para satisfacer este interés variarán de acuerdo a la cultura política de cada sociedad.

\subsection{La Democracia Internacional como derecho de los Estados}

A diferencia de lo que sucede a nivel doméstico, en donde un sistema democrático — dada la cultura política pública - puede encontrarse fundado en consideraciones

52 Al tratar este interés BEITZ oscila entre considerarlo como uno de índole igualitaria o suficientaria. Así, señala: «...(i)n the extreme case, when some people are excluded entirely from any public role (as, for example, with the wholesale denial of the franchise to blacks in the antebellum South), it has been said that those excluded 'are not publicly recognized as persons at all' and may be described as "socially dead"...». Hasta aquí su posición parece suficientista, pero luego agrega «...(s) omething similar occurs when procedural roles are assigned in a way that coveys social acceptance of a belief in the inferiority or lesser merit of one group... because it is a fixed point in a democratic culture that public institutions should not establish or reinforce the perception that some people's interests deserve less respect or concern than those of others...» (BEITZ, 1990: 109-110). En mi opinión, esta última exigencia de igual tratamiento es un requerimiento de justicia, no de legitimidad.

${ }_{53}$ Una vez más, BEITz mismo oscila entre el igualitarismo y el suficientismo al tratar este interés. Su posición parece ser igualitaria cuando sostiene: «...Political decisions could then be said to satisfy the interest in equitable treatment when, over time, they promote (or do not systematically detract from) a distribution that accords with the requirements of justice, which are themselves to be worked out from a point of view in which each person's prospects are taken equally into account» (BEITZ, 1990: 112). Pero más tarde, parece inclinarse por el suficientismo cuando afirma que «...in the context of reasoning about political procedures, the interest in equitable treatment will normally appear as an interest in safeguarding one's urgent or vital interests in the face of the threat that they might be systematically subordinated to the competing but less urgent claims of others...» (BEITZ, 1990: 113).

54 BEITZ parece concebir las demandas de satisfacer este interés como si fuesen suficientistas. Sostiene que «...deliberation should not be constrained by the exclusion of positions that would gain substantial support if they were sufficiently exposed to public scrutiny...» (BEITZ, 1990: 114). Pueden encontrarse vacilaciones en GUTMMAN y THOMPSON, cuando discuten el modo en que la democracia deliberativa puede corregir los sesgos: «...To the extent that the least advantaged are excluded because they are too poor to have equal access to the political media, the principles of deliberative democracy support an effective critique of this unfairness...» (GUTMMAN y THOMPSON, 2004: 48). No está claro si el problema radica en la exclusion —y las demandas insatisfechas son suficientarias - o en la desigualdad. Hasta Th. CHRIsTIANO, quien adopta una posición claramente igualitaria con respecto a la legitimidad, apela a argumentos suficientistas: «...Each person has an interest in being taken seriously by others. When an individual's views are ignored or not given any weight, this undermines his sense of self-respect...» (CHRISTIANO, 1997: 259). 
que no pueden ser vistas como razones por aquellos a quienes se aplica, a nivel internacional sólo un procedimiento democrático es apto para satisfacer el interés deliberativo de los Estados. Dicho en otras palabras, el interés que poseen los individuos organizados políticamente, en que el sistema de toma de decisiones internacional sea sensible a sus opiniones sólo puede ser satisfecho si el procedimiento adoptado es de índole democrático. La razón de esta asimetría subyace en el hecho de que una de las ideas centrales de la cultura política pública internacional es que los Estados deben ser tratados como libres e iguales. Mientras a nivel doméstico existen sociedades donde la idea de ciudadanía libre e igual no se encuentra implícita en la cultura política, a nivel internacional tal extremo se encuentra configurado.

Aun en aquellas sociedades donde no se considera que los ciudadanos deban ser tratados como libres e iguales, se piensa que en tanto cuerpo de individuos políticamente organizados deben ser tratados en la arena internacional por otros Estados como libres e iguales. Individuos que dada su cultura política doméstica no consideran que sus conciudadanos deban ser tratados como libres e iguales, sí consideran que su Estado - esto es ellos mismos en tanto organizados políticamente junto con sus conciudadanos- debe ser tratado como libre e igual. Así, por ejemplo, condenan la interferencia extranjera en los asuntos domésticos de su Estado, o promueven que su Estado sea escuchado como un igual cuando se trata de adoptar resoluciones sobre cuestiones internacionales.

Dado que éste es el caso, es posible afirmar que existen ciertos rasgos del actual orden internacional que no satisfacen los intereses de autoría que poseen los Estados. Así, por ejemplo, el procedimiento de toma de decisiones colectivas corporizado en el diseño institucional del Consejo de Seguridad, no satisface el interés que los Estados - en tanto autores de las instituciones internacionales - tienen en la responsabilidad deliberativa. El diseño otorga más peso a las opiniones de algunos Estados, los cinco miembros permanentes con derecho a veto: Estados Unidos, Gran Bretaña, Rusia, China y Francia. Un esquema institucional semejante no trata como autores a todos los Estados a quienes se aplica coercitivamente. Aunque las opiniones de todos acerca de cómo conducir los asuntos colectivos pueden ser consideradas — por ejemplo, porque los miembros permanentes alinean sus decisiones con las opiniones de la mayoría- el procedimiento mismo de toma de decisiones no se encuentra fundado en razones que sean aceptables para todos a partir de las ideas implícitas en la cultura política pública internacional. Un procedimiento de decisión que confiere poder de veto a algunos Estados no se encuentra fundado en consideraciones que los Estados — dada la cultura política pública internacional y su idea implícita de libertad e igualdad - puedan ver como razones.

Adicionalmente, un sistema de toma de decisiones semejante no satisface el interés en el reconocimiento que cada Estado posee. Lo que determina que esto sea así es que algunos Estados no pueden ocupar el rol de miembros permanentes. No se trata de que sus opiniones no son consideradas, sino de que ellos ocupan cierta posición pública de inferioridad en el esquema institucional que quedó configurado luego de la Segunda Guerra Mundial. La razón de esta exclusión simplemente refleja la opinión de que se trata de Estados débiles. Mientras los ganadores y poderosos se reservaron los lugares permanentes, al resto sólo le fue concedido acceder a los puestos rotativos. 
En síntesis, que existan miembros permanentes y no permanentes vulnera el interés en el reconocimiento, que los miembros permanentes tengan derecho a veto vulnera el interés en la responsabilidad deliberativa.

Lo que muestra el ejemplo del Consejo de Seguridad, es que aunque existe un derecho a la democracia que el esquema de instituciones internacionales debe garantizar, no se trata de un derecho humano que cada uno de los ciudadanos tenga respecto de su propio esquema de instituciones estatales, sino de un derecho que los ciudadanos políticamente organizados - los Estados legítimos- tienen con respecto al esquema internacional. No existe un Derecho Humano a la Democracia - que sea la consecuencia de la aplicación coercitiva del sistema de Estados por parte del orden internacional sobre los individuos - sino un derecho estatal a la democracia que se sigue de la aplicación coercitiva del orden internacional sobre los Estados.

La existencia de un derecho a la Democracia Internacional, no sólo sirve para criticar las instituciones existentes como el Consejo de Seguridad, sino también para defenderlas de algunas críticas usuales. Permite advertir que ciertos rasgos del orden internacional que usualmente son considerados problemáticos, no son tales. Me refiero específicamente a la inexistencia de un demos global, de un órgano representativo elegido directamente por la población mundial, o de nuevos modos de permitir el control de las instituciones internacionales con respecto al demos global ${ }^{55}$. Muchos de quienes adscriben a un paradigma democrático de legitimidad política, consideran que estos rasgos constituyen un déficit democrático del actual orden internacional. La concepción que he presentado, ayuda a entender porqué esto no es así. Si las exigencias de legitimidad democrática internacional son el correlato de la imposición coercitiva del orden internacional sobre los Estados, son éstos y no las personas individualmente consideradas las unidades políticamente relevantes. No sólo no es un déficit que la representación internacional esté mediada por los Estados sino que es moralmente requerido que así sea. Si quienes son coercionados son los Estados, son sus intereses de autoría los que deben satisfacerse y, por ende, son los Estados quienes deben estar representados en los órganos de decisión internacional.

Puesto de modo concreto, que en la Asamblea de las Naciones Unidas estén representados los Estados y no los individuos es una virtud a ser potenciada y no un defecto que deba ser remediado ${ }^{56}$. De lo que se trata no es de modificar el tipo de representación sino, por un lado, de aumentar el control que la Asamblea tiene sobre las decisiones que sirven para configurar las instituciones internacionales y, por el otro, de garantizar que el procedimiento de toma de decisiones sea de índole democrática.

55 Quienes como J. COHEN y Ch. SABEL (2005) sostienen que existe un nuevo poder coercitivo global que se aplica directamente sobre los individuos también sostienen que es necesario que se instauren nuevos mecanismos de global accountability.

56 Algunas propuestas de reformas cosmopolitas, se acomodan a la concepción que he presentado. Así, la idea de D. HeLD (2006: 306) de conformar una Asamblea de las Naciones Unidas dotada de autoridad sobre ciertos temas que conciernen globalmente, es perfectamente adecuada a lo que he señalado en el texto. Otras propuestas, como la de llevar adelante referéndums a nivel global o regional, son más difícil de acomodar a la visión que he presentado en el texto (HELD, 2006: 307). Si los resultados de estas consultas no son ponderados de tal modo que den cuenta de la existencia de diversas comunidades políticas sobre las que se aplican diversos órdenes coercitivos, su utilización no se encuentra justificada. 


\section{INTERVENCIÓN HUMANITARIA Y AUTOGOBIERNO}

La concepción general de los derechos humanos que he presentado tiene profundas repercusiones prácticas en lo que respecta a la intervención humanitaria. En primer lugar, sirve para vislumbrar dónde reside el error de la política estadounidense y cómo debería ser enmendado. El mandato democratizador, eje de la política exterior de los últimos años, no debería ser entendido como dirigido a instaurar regímenes domésticos democráticos allí donde no existen, con total independencia de las características de la cultura política pública de la sociedad intervenida. Las políticas de nation building llevadas adelante por los países occidentales - uno de cuyos objetivos ha sido la instauración de regímenes democráticos- en sociedades como la afgana cuyas culturas no poseen implícita la idea de ciudadanía libre e igual, no están moralmente justificadas ${ }^{57}$.

La política exterior de los Estados occidentales en relación con aquellas sociedades que no tienen implícita la idea de ciudadanía libre e igual, como presumiblemente son algunas sociedades islámicas, debe adoptar el rumbo que caracterizó la política estadounidense con respecto a estas sociedades durante la guerra fría ${ }^{58}$. Durante este periodo se acuño la idea del «excepcionalismo árabe» que determinaba que la promoción de la democracia - que era una política válida con respecto a otros Estados- no lo fuese con respecto a ciertos países islámicos. No obstante, aunque este «excepcionalismo» es adecuado, deben alterarse las razones utilizadas para justificarlo. Durante la guerra fría las razones que determinaron esta directriz de la política exterior estadounidense fueron meramente estratégicas ${ }^{59}$. El argumento que he ofrecido muestra que existen

57 A pesar de que la cultura política de la mayor parte de los países islámicos no es democrática —no alberga la idea de ciudadanía libre e igual- existen datos que dan razones para pensar que tal situación está cambiando. Según The Pew Global Attitudes Survey del 2005: «...A plurality of Lebanese (46\%) —and a majority of the country's Christians (59\%) - are optimistic that the Middle East will become more democratic. Meanwhile, Jordanians are divided between those who are becoming more optimistic and pessimistic, while the Turks, Pakistanis, and Moroccans lean toward pessimism (although 34\% of Pakistanis offer no opinion)» (PEW GLOBAL AtTitudes Project, 2005). Aunque parece haber una correlación entre el carácter predominantemente cristiano de la población y sus actitudes positivas hacia la democracia, también en los países de mayoría musulmana la situación es cambiante. Así, por ejemplo, según la encuesta del año 2002, el 63 por 100 de los jordanos creían que la democracia no era una forma de gobierno característicamente occidental. En el año 2005 esta cifra se había elevado al 80 por 100.

58 La ausencia de un Derecho Humano a la Democracia, sin embargo, no convalida la política que Estados Unidos ha llevado adelante en medio oriente brindando soporte activo o tolerando regímenes autocráticos. La política exterior estadounidense ha tenido aquí por objetivo promover la estabilidad, aun a costa del sacrificio de los derechos humanos. Al respecto señala T. C. WITTES: «Stability there is necessary to ensure the free flow of oil and gas through the Persian Gulf to world markets, to facilitate the movement of U.S. naval and commercial traffic from the Mediterranean to the Indian Ocean via the Suez Canal, and to protect the security of key regional allies, including the State of Israel and the Kingdom of Saudi Arabia...» (WITTES, 2008: 16). Aunque no existe un Derecho Humano a la Democracia, existe un derecho a que los regímenes políticos sean sensibles a las opiniones de los ciudadanos que Estados Unidos no ha ayudado a satisfacer.

59 WITTE resume las razones detrás de la política exterior estadounidense con respecto a los países árabes durante la guerra fría del siguiente modo: «First, there is the concern, widespread among U.S. policymakers, that putting a greater emphasis on democracy in America's dialogue with Arab regimes will necessarily bump other issues down the priority list or even demand trade-offs. Second, there is the belief that U.S.-Arab cooperation, despite being rooted in mutual self-interest, is fragile and that pressure on Arab governments to yield power at home will lead those governments to loosen or even abandon their strategic alliances with Washington» (WITTE, 2008: 18). 
razones de principio para no promover en ciertos países la instauración de regímenes democráticos. Lo que justifica el «excepcionalismo» es la idea de que instalar regímenes democráticos en ciertas sociedades no promueve el auto-gobierno.

No obstante, el mandato democratizador debe continuar siendo uno de los ejes de la política exterior de los Estados occidentales. El argumento que he ofrecido no muestra que debe ser abandonado por completo sino que debe ser redireccionado. No debe tener por objeto garantizar un Derecho Humano a la Democracia a través de la promoción de regímenes estatales democráticos. Lo que debe ser garantizado es un derecho a la Democracia Internacional a través de la promoción de instituciones internacionales más igualitarias. Los Estados deben trabajar activamente para que las instituciones democráticas como la Asamblea de la ONU tenga más incidencia en la toma de decisiones internacionales y para democratizar aquellas instituciones internacionales que poseen un sesgo anti-democrático, como es el caso del Consejo de Seguridad de Naciones Unidas.

Por lo que respecta a la reforma de instituciones con sesgo antidemocrático, un ejemplo de los cambios concretos que deberían promover los Estados puede encontrarse en la resolución 1991 de la Asamblea XVIII de Naciones Unidas. La medida entró en vigor el 31 de agosto de 1965, y aumentó el número de los miembros no permanentes que pasaron de seis a diez. Aunque esta reforma aumentó la calidad democrática del proceso de toma de decisiones en el seno del Consejo, no alteró la distinción entre miembros permanentes y no permanentes o el derecho a veto de los primeros. En este sentido la reforma fue positiva y necesaria, aunque no fue suficiente.

La segunda directriz que deben perseguir los Estados es la de dar preeminencia a la hora de tomar decisiones a las instituciones internacionales democráticas actualmente existentes. Las líneas de trabajo para lograr tal cometido son dos. Primero, debería promoverse que los representantes de los Estados en los órganos internacionales —paradigmáticamente la Asamblea General de la ONU— se encuentren bajo el control de los órganos estatales dotados de mayor representatividad o legitimidad política. Si el Estado del que se trata es una democracia representativa, este órgano será el parlamento ${ }^{60}$. No obstante, como es posible que otras formas de organización política sean legítimas la dependencia del parlamento no debe considerarse como el único modo de satisfacer esta exigencia. Segundo, debería promoverse que el grueso de las decisiones internacionales estuviese en manos de los órganos internacionales más representativos ${ }^{61}$. Mientras más número de las competencias internacionales se atribuyan a la Asamblea General, o mientras más control ésta ejerza sobre las otras agencias internacionales, mayor será la legitimidad política internacional que existe.

Lo señalado con respecto al mandato democratizador se aplica con especial fuerza a la institución internacional de la intervención humanitaria. En primer lugar, la existencia de un derecho Internacional a la Democracia, ofrece una guía para responder a la pregunta de cómo debería decidirse la Intervención. Tal decisión debería ser

${ }^{60}$ Aun si actualmente es difícil encontrar sistemas legítimos no democráticos, es necesario por las razones ofrecidas en el texto dejar abierta esta posibilidad.

${ }^{61}$ Que un número creciente de decisiones a nivel internacional sea dejada en mano de grupos conformados por miembros de la rama ejecutiva de gobierno - tales como el G20 o el G7 — debe ser visto como rasgo problemático del actual orden internacional. 
adoptada por la comunidad internacional — debido a que ella es la garante de que los Estados respeten los derechos humanos de sus ciudadanos- a través de un proceso democrático — debido a que de lo contrario la decisión carecería de legitimidad-. Sólo en este caso la decisión de intervenir satisface las exigencias de legitimidad que se aplican al esquema internacional. Así, puede afirmarse que el creciente número de intervenciones unilaterales, llevadas adelante por Estados Unidos y otras potencias militares, con total independencia de lo loable que hayan sido sus objetivos, carecen de legitimidad moral ${ }^{62}$.

Adicionalmente, la concepción que he presentado también ayuda a responder a la pregunta acerca de cuándo está justificada la intervención humanitaria y por qué. Con respecto al cuándo, si un Estado no tiene la capacidad o la voluntad de respetar los derechos humanos de sus ciudadanos, entonces la Comunidad Internacional tiene la responsabilidad - y el derecho- de intervenir. En este supuesto — dando por sentado que la intención de la intervención es acabar con la violación de los derechos humanos, todos los medios pacíficos han sido agotados, los medios utilizados son proporcionales y existe una perspectiva razonable de éxito- la intervención se encuentra justificada.

En relación con el porqué la respuesta tiene dos partes. En primer lugar, la Comunidad Internacional tiene el deber de intervenir porque sobre ella recae la responsabilidad de garantizar que los Estados respeten los derechos humanos de sus ciudadanos. Que el orden internacional imponga de modo coercitivo sobre todos los seres humanos un sistema de Estados, es el origen de esta responsabilidad. En segundo lugar, la Comunidad Internacional tiene el derecho de intervenir —o lo que es lo mismo, los Estados no tienen derecho a oponerse a la intervención- porque si los derechos humanos no son satisfechos, desaparece la barrera moral que el respeto por el autogobierno impone sobre los actos de intervención.

En síntesis, que existan dos órdenes coercitivos de diferente nivel —el internacional y el doméstico — con sus correlativas exigencias de legitimidad, sirve para explicar, por un lado, cómo debe adoptarse la decisión de intervenir y, por el otro, cuándo y porqué la intervención humanitaria se encuentra justificada. Que el orden internacional se aplique de modo coercitivo sobre los Estados — y las exigencias de legitimidad y Democracia Internacional que esto engendra - es relevante para establecer cómo decidir la intervención. Que las instituciones estatales se apliquen de modo coercitivo sobre los ciudadanos - y las exigencias de legitimidad que esto engendra - es relevante para establecer cuándo intervenir. Que el orden internacional imponga sobre los seres humanos un sistema de Estados y que se aplique coercitivamente a esos mismos Estados $-\mathrm{y}$ las exigencias de legitimidad que esto engendra - es relevante para establecer por qué la Comunidad Internacional tiene el derecho y el deber, respectivamente, de intervenir.

Finalmente, aunque el argumento que he ofrecido en el texto no permite invocar el Derecho Humano a la Democracia para mostrar que existe un caso de intervención

${ }^{62}$ La intervención internacional en Irak por parte de Estados Unidos en el año 2003 es un caso paradigmático de intervención ilegítima. Lo que provocó revuelo internacional no fue la intervención — desde el final de la década de los ochenta las intervenciones armadas autorizadas por las Naciones Unidas se habían incrementado sustancialmente- sino su carácter unilateral. 
humanitaria que no vulnera el auto-gobierno, posibilita ofrecer una respuesta mucho más contundente a esta objeción. Contrario a la posición defendida por el gobierno chino, permite mostrar que ningún tipo de intervención humanitaria $-\mathrm{y}$ no sólo la fundada en un Derecho Humano a la Democracia - vulnera el auto-gobierno. Las razones que fundan esta conclusión son tres.

En primer lugar, según la concepción que he presentado el interés en el autogobierno es uno que poseen los individuos a quienes se aplica coercitivamente el esquema de instituciones estatales. No se trata de un interés que posean los Estados. Esto permite desarticular la equivalencia entre el interés de los individuos en el autogobierno y el derecho de los Estados a la no-intervención. No todo Estado tiene derecho a la no-intervención sino sólo aquellos en los que el interés de los individuos en el auto-gobierno se encuentra satisfecho. No basta constatar un acto de intervención para sostener que el interés en el auto-gobierno de los ciudadanos ha sido vulnerado.

En segundo lugar, puesto que he defendido una posición que concibe a la satisfacción de los derechos humanos como condición necesaria y conjuntamente suficiente para que exista legitimidad o auto-gobierno, nunca puede darse el caso que la intervención humanitaria vulnere el valor del auto-gobierno. Si lo que justifica la intervención humanitaria es la existencia de violaciones a los derechos humanos, allí donde la intervención está moralmente justificada no existe esquema institucional legítimo ni auto-gobierno que sea vulnerado.

En tercer lugar, concebir a los derechos humanos como he propuesto permite advertir que el objetivo que persigue toda intervención humanitaria es promover la existencia de auto-gobierno o legitimidad política. Si los derechos humanos son condiciones necesarias y suficientes de legitimidad, y el objetivo de la intervención humanitaria es garantizar su satisfacción, se sigue que su objetivo también es promover el auto-gobierno o la legitimidad. Cualquier intervención que tenga por objeto poner fin a la vulneración de los derechos humanos no puede implicar costo alguno en términos de auto-gobierno, dado que su objetivo es precisamente promover las condiciones que deben darse para que el auto-gobierno exista.

\section{CONCLUSIÓN}

La concepción de los derechos humanos que he presentado parte de la constatación que tanto el esquema de instituciones internacionales como domésticas poseen carácter coercitivo. Las exigencias morales que trae aparejada la existencia de esquemas coercitivos son las de autoría o legitimidad política. Dado que las instituciones internacionales y las domésticas son coercitivas en diferente forma y sobre diferentes sujetos, las exigencias de autoría que la existencia de cada una engendra son diferentes. $\mathrm{La}$ existencia de instituciones estatales trae aparejado que a los individuos les deban ser conferidos ciertos derechos, oportunidades y recursos. Las instituciones que satisfacen estas exigencias ubican a los individuos a quienes se aplican coercitivamente en el rol de autor y son, por ende, legítimas. La existencia de instituciones internacionales que poseen carácter coercitivo tanto sobre los individuos como sobre los Estados trae aparejado que a unos y otros les deban ser conferidos por éstas ciertos derechos, 
oportunidades y recursos. Por lo que respecta a los individuos, las instituciones internacionales deben ser garantes de que los Estados en los que habitan los ubiquen en el rol de autores confiriéndoles los derechos, oportunidades y recursos necesarios. La institución de los derechos humanos ha sido la herramienta elegida por el orden internacional para volver efectiva esta garantía. Por lo que respecta a los Estados, las instituciones internacionales deben conferirles los derechos, oportunidades y recursos necesarios para que sus intereses de autoría se encuentren satisfechos. Así como las instituciones domésticas deben ubicar en el rol de autores a los ciudadanos, las instituciones internacionales deben ubicar en el rol de autores a los Estados, esto es, a los individuos políticamente organizados.

Lo señalado trae aparejado que, dadas las peculiaridades de las diferentes culturas políticas públicas domésticas y de la cultura política pública internacional, aunque no existe un Derecho Humano a la Democracia — puesto que pueden existir Estados legítimos no-democráticos- sí existe un derecho a la Democracia Internacional — puesto que sólo un orden internacional democrático es legítimo- - Constatar tal extremo trae profundas consecuencias para el diseño y la justificación moral de la institución de la intervención humanitaria. Un nuevo mandato democratizador cobra vigencia. Uno que no se refiere a la democratización de las instituciones estatales, sino a la democratización de las instituciones internacionales.

\section{BIBLIOGRAFÍA}

BeITZ, Ch., 1979-1999: Political Theory and International Relations, Princeton: Princeton University Press.

- 1990: Political Equality: An Essay in Democratic Theory, Princeton, NJ: Princeton University Press.

- 2009: The Idea of Human Rights, Oxford: Oxford University Press.

Blake, Ch., 2002: «Distributive Justice, State Coertion, and Autonomy», Philosopby and Public Affairs, 30, 257-296.

BuCHANAm, A., 2004: Justice, Legitimacy and Self-determination: Moral Fundations for International Law, Oxford: Oxford University Press.

BusH, G. W.: http://stateoftheunion.onetwothree.net/texts/20040120.btml.

Chomsky, N., 1992: Deterring Democracy, New York: Verso.

Christiano, Th., 1997: «The Significance of Public Deliberation», en J. BoHman y W. REHG (eds.), Deliberative Democracy: Essays on Reason and Politics, Cambridge, Massachusetts: The MIT Press, 243-277.

Cohen, G., 2000: If You're an Egalitarian, How Come You're So Rich?, Cambridge, Mass.: Harvard University Press.

COHEN, J., y SABEL, Ch., 2005: «Global Democracy?», NYU Journal of International Law and Politics, vol. 37, núm. 4, 763-797.

Donelli, J., 1989: Universal Human Rights in Theory and Practice, Ithaca: Cornell University Press.

FrANCK, T. M., 1992: «The Emerging Right to Democratic Governance», The American Journal of International Law, vol. 86, 46-91.

Fox, G., 1992: «The Right to Political Participation in International Law», Yale Journal of International Law, vol. 7, 579-590. 
Gutmman, A., y Thompson, D., 2004: Why Deliberative Democracy, Princeton, New Jersey: Princeton University Press.

Held, D., 2006: Models of Democracy, 3. ${ }^{\text {a }}$ ed., Cambridge: Polity Press.

Hook, S. W., 2002: «Inconsistent U.S. Efforts to Promote Democracy Abroad», en P. J. SCHRAEDER (ed.), Exporting Democracy; Rhetoric vs. Reality, London \& Boulder, CO: Lynne Rienner, 109-128.

ICISS, 2001: The Responsibility to Protect: Report of the International Commission on Intervention and State Sovereignty, Ottawa: International Development Research Centre.

Karmen, A., 2000: «Poverty, Crime and Criminal Justice», en HefFernan, W., y Kleining, J. (eds.), From Social Justice to Criminal Justice. Poverty and the Administration of Criminal Law, Oxford: Oxford University Press, 25-46.

Kent, A., 1993: Between Freedom and Subsistence. China and Human Rights, Hong Kong: Oxford University Press.

Kingsbury, B.; Krisch, N., y Stewart, R., 2005: «The Emergence of Global Administrative Law», Law E Contemporary Problems, 68, 15-61.

KiRKPATRICK, J., 1979: «Dictatorships and Double Standards», Commentary Magazine, vol. 68, núm. 5, 34-45.

Luban, D., 1980: «Just War and Human Rights», Philosophy and Public Affairs, 9, 160-181.

- 2002: «Intervention and Civilization: Some Unhappy Lessons of the Kosovo War», en P. DE Greiff y C. Cronin (eds.), Global Justice and Transnational Politics, Cambridge, Mass.: MIT Press.

MANSFIELd, E. D., y SNYDER, J., 2005: Electing to Fight: Why Emerging Democracies Go to War, Cambridge, Mass.: MIT Press.

Moellendorf, D., 2002: Cosmopolitan Justice, Colorado: Westview Press.

MuravchiK, J., 1991: Exporting Democracy. Fulfilling America's Destiny, Washington: American Eterprise Institute Press.

NeIER, A., 1997: «The New Double Standard», Foreign Policy, 105, 91-102.

ONU, 2005: World Summit Outcome, A/RES/60/1, http://www.un.org/summit2005/documents. btml (última visita, mayo de 2012).

PetTit, Ph., 2008: Made with Words: Hobbes on Language, Mind and Politics, New Jersey: Princeton University Press.

Pettit, Ph., y Lovett, F., 2009: «Neorepublicanism: A Normative and Institutional Research Program», Annual Review of Political Science, 12, 11-29.

— 2009a: «Law and Liberty», en Besson, S., y MARTí, J. L. (eds.), Legal Republicanism: National and International Perspectives, Oxford: Oxford University Press, 39-59.

- 2010: «Legitimate International Institutions: a Neo-republican Perspective», en BESSON, S., y Tasioulas, J. (eds.), The Philosophy of International Law, Oxford: Oxford University Press (en prensa).

Pew Global Attitudes Project, 2005: «How the United States is Perceived in the Arab and Muslim Worlds», noviembre de 2005, http://www.pewglobal.org/2005/11/10/how-the-united-states-is-perceived-in-the-arab-and-muslim-worlds/.

Pogge, Th., 1989: Realizing Rawls, Ithaca: Cornell University.

RawLs, J., 1993: Political Liberalism, New York: Columbia University Press.

- 1999: The Law of Peoples, Cambridge, Massachusetts: Harvard University Press.

Ripstein, A., 2004: «Authority and Coercion», Philosopby and Public Affairs, 32, 2-35.

Roosvelt, F. D., 1940: Address of December 29, 1940, http://www.presidentialrbetoric.com/ historicspeeches/roosevelt_franklin/arsenal.html. 
SCAnlon, T. M., 1998: What We Owe to Each Other. Cambridge, Mass.: Belknap Press.

Seleme, H. O., 2007: «Legitimidad Política, Justicia y Globalización», Revista Argentina de Teoría Jurídica, 9, 1-60.

- 2009: «La consistencia del contextualismo institucional e internacional», Enrahonar, 43, 205-228.

- 2010: «La legitimidad como autoría», Revista Brasileira de Filosofía, vol. 234, año 59, 73-99.

Tesón, F., 1998: A Philosophy of International Law, Colorado: Westview Press.

VinCENT, J. R., 1974: Nonintervention and International Order, Princeton: Princeton University Press.

Wilson, W., 1917: War Message to Congress (2 de abril de 1917), bttp://wwi.lib.byu.edu/index. php/Wilson's_War_Message_to_Congress.

Williams, B. (1981): «Internal and External Reasons», en Moral Luck, Cambridge: Cambridge University Press.

Wittes, T. C., 2008: Freedom's Unsteady March: America's Role in Building Arab Democracy, Washington, D. C.: Brrokings Institution Press. 